WIENER SLAVISTISCHES JAHRBUCH, Band 56/2010, 39-60

(C) 2010 by Österreichische Akademie der Wissenschaften, Wien

GEORG HOLZER

$\mathrm{S}$ A ŠA D OBRIĆ

E T A L I I

\title{
Lautgeschichtliches Glossar zum Neuštokavischen
}

Dieser Beitrag ist das Ergebnis der Arbeit im von Georg Holzer geleiteten Seminar, das im Vorlesungsverzeichnis der Universität Wien unter der Nummer 130445 und dem Titel „Linguistisches Seminar - für Bosnisch/Kroatisch/Serbisch“ geführt und im Sommersemester 2008 abgehalten wurde. Holzer selbst hat alle hier angeführten urslavischen Lautungen rekonstruiert und ihre neuštokavischen Outputs hinzugefügt. Auch alle über die Ableitungsschritte hinausgehenden Textelemente und Anmerkungen stammen von ihm. Die Aufgabe der Teilnehmerinnen und Teilnehmer am Seminar - ihre Namen lauten in alphabetischer Reihenfolge: Marin B e r la kovich, Saša Dobrić, Magdalena Đuričić, Olga Joachimsthal, Sonja Joks imović, Marija Pavlović, Emina Pičorušević, Dragica Saraf, Mirjana S araf, Adela Sarvan, Ifet Sivić, Gordana Staji ć, Romana Š i ši ć - war es, anhand von Holzer 2007 den lautgeschichtlichen Werdegang der einzelnen lexikalischen Einheiten zwischen ihrer urslavischen und ihrer neuštokavischen Lautung nach dem Vorbild des Glossars in Holzer 2007: 90-144 darzustellen, also die mit Ziffern bezeichneten einzelnen lautgeschichtlichen Etappen ab der ersten nachurslavischen Lautung anzuführen. Die Ziffern entsprechen denen, welche in Holzer 2007: 51-88 die einzelnen Lautgesetze in chronologisch gemeinter Reihenfolge nummerieren. Somit stellt die vorliegende Arbeit eine Ergänzung zum Glossar in Holzer 2007 dar. Jeder Teilnehmer und jede Teilnehmerin hatte ihm bzw. ihr zugeteilte Einträge zu bearbeiten; die einzelnen Einträge sind hier mit den betreffenden Namen versehen. Während des Seminars wurden die Ergebnisse in Referaten vorgetragen und dabei Fehler korrigiert. S a š a D o bri ć hat dann seine Arbeit und die Arbeiten der anderen Teilnehmer und Teilnehmerinnen zusammengetragen, zum hier vorliegenden alphabetisch geordneten Glossar vereinigt und von ihm entdeckte übrig gebliebene Fehler korrigiert. Danach hat der Seminarleiter weitere Korrekturen vorgenommen und die Literaturhinweise und die Literaturliste hinzugefügt sowie alle zusätzlichen Anmerkungen angebracht. 
$\mathrm{Zu}$ den Notationen urslavischer und neuštokavischer Wörter s. Holzer 2007: 13 und 16-19.

In Holzer 2009: $165 \S 11.5$ ist in Abänderung der in Holzer 2007: 56 f. gegebenen Reihung die Meilletsche Metatonie (11) nach dem Wirken des Dyboschen Gesetzes (12) angesetzt; diese Korrektur ist hier in der Bezifferung der Lautgesetze noch nicht berücksichtigt; auf die Reihenfolge der lautgeschichtlichen Etappen eines Wortes wirkt sich die Änderung jedoch nicht aus, weil Silben, die Meillets Metatonie unterlagen, nicht auch von Dybos Gesetz erfasst werden konnten und umgekehrt. In Holzer 2010: 52 ist ursl. *bajsu 'Dämon' angesetzt; das hat Holzer hier s. v. bijês auf ursl. *baj su korrigiert.

bäba: ursl. * $b \underline{a} \cdot b \bar{a} \cdot>31 * b \underline{a} \cdot b a>{ }_{43}$ nštok. $b \underline{a} b a$ 'Großmutter' (Šišić). Vgl. Skok I 82, Snoj 2003: 27, Derksen 2008: 32.

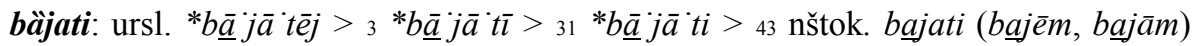
'beschwören, zaubern' (Đuričić). Vgl. Skok I 93, Snoj 2003: 27 f., Derksen 2008: $33 \mathrm{f}$.

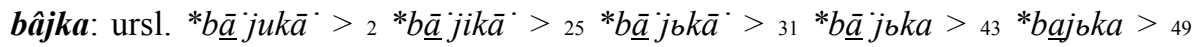
*bajka > 67 nštok. bāj kka 'Märchen' (Dobrić). Vgl. Skok I 93, Snoj 2003: 28.

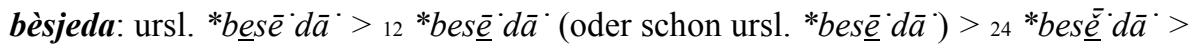

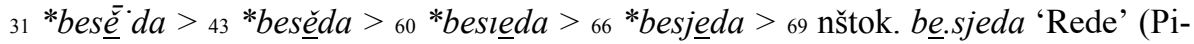
čorušević). Vgl. Skok I 140 f., Snoj 2003: 37.

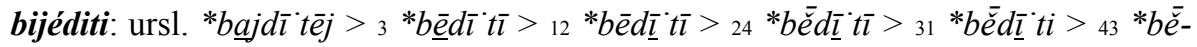

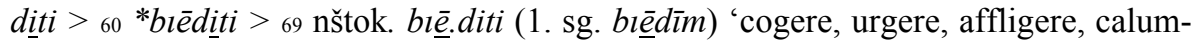
nari’ (Đuričić). Vgl. ARj I 291, Skok I 148 f., Derksen 2008: 39.

bijês: ursl. *baj $s u>{ }_{3} * b \bar{e} s u>{ }_{11} * b \bar{e} s u>{ }_{24} * b e \check{s} s u>{ }_{25} * b e \check{s} s b>30 * b \underline{e} s b>39 * b \underline{e} s b$ $>{ }_{9} * b \underline{\underline{e}} s>60$ nštok. blēs 'Wut' (Šišić). Vgl. Skok I 149, Snoj 2003: 37, Derksen 2008: 40.

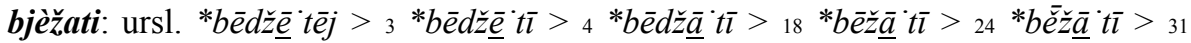

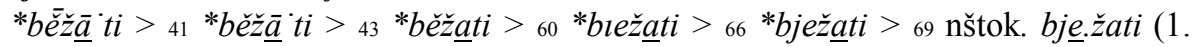
sg. bje.žim) 'fliehen' (Pičorušević). Vgl. ARj I 391, Skok I 166, Snoj 2003: 39, Derksen 2008: $40 \mathrm{f}$.

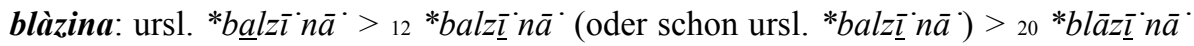

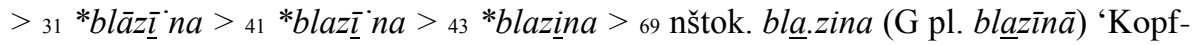
polster' (M. Saraf). Vgl. ARj I 427, Skok I 171, Snoj 2003: 45, Derksen 2008: 54. Zum Suffix s. Holzer 2010: $51 \mathrm{f}$.

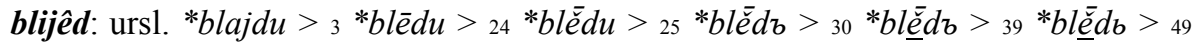

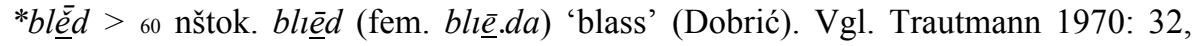
Skok I 174, Snoj 2003: 45, Derksen 2008: 42 f. 


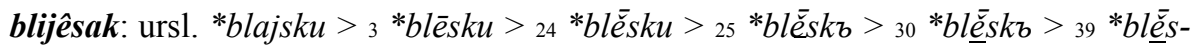
$k b>{ }_{49} *$ ble $\underline{e} s k>60$ nštok. *bliēesk, daraus durch Analogie bliēsak 'Blitz' (Pičorušević). Vgl. Skok I 174 f., Snoj 2003: 46, Derksen 2008: 43.

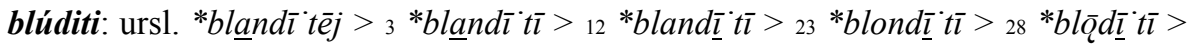

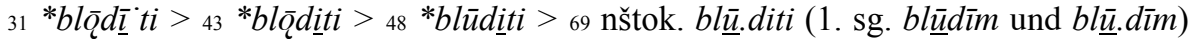
'irren, sich verirren’ (M. Saraf). Vgl. ARj I 451 f., Skok I 173 f., Snoj 2003: 47, Derksen 2008: 47.

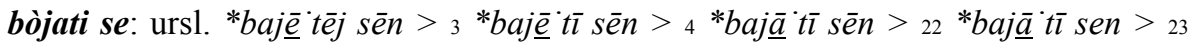

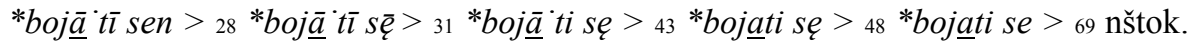
bo.jati se (1. sg. bo.jīm se) 'sich fürchten' (Dobrić). Vgl. Skok I 183, Snoj 2003: 33, Derksen 2008: 50. Zum Reflexivpronomen se vgl. me in Holzer 2007: 114.

bôk: ursl. ${ }^{*} b a k u>{ }_{23} * b o k u>{ }_{25} * b o k b>{ }_{29} * b \bar{o} k b>{ }_{30} * b \underline{o} k b>39 * b \underline{o} k b>49$ nštok. $b \underline{\underline{\alpha} k}$ (G sg. bokka) 'Hüfte' (M. Saraf). Vgl. Skok I 183, Snoj 2003: 50.

böljī: ursl. *balijuju $>_{2} *$ balijiji $>{ }_{12} *$ balijiji $>_{23} *$ bolijiji $>{ }_{25} *$ bolbjbjb $>{ }_{32} *$ bolbji

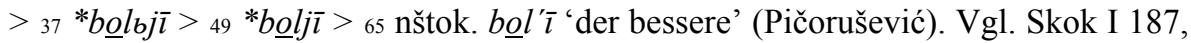
Snoj 2003: 50 s. v. bölj, Derksen 2008: 52.

bôs: ursl. *basu $>{ }_{23} *$ bosu $>{ }_{25} *$ bosb $>29 * b \overline{o s b}>{ }_{30} * b \underline{o} s b>39 * b \underline{o} s b>49$ nštok. bōs (fem. bo.sa) 'barfuß’ (Đuričić). Vgl. Skok I 191, Snoj 2003: 52, Derksen 2008 : $59 \mathrm{f}$.

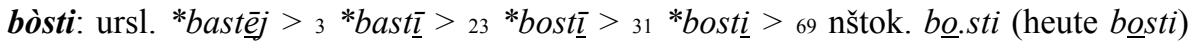
'stechen' (Dobrić). Zur Akzentuierung des Infinitivs s. Holzer 2007: 118 Fußnote 49. Vgl. Skok I 191 f., Snoj 2003: 52, Derksen 2008: 59, Holzer 2010: 52 f. Nach Dybo 1981: 208 konjugieren von den $e$-Verben mit Wurzeln auf Obstruent alle bis auf wenige von ihm genannte nach dem Akzentparadigma c.

bráda: ursl. *bard $\underline{a}^{*}>{ }_{20} * b r a \bar{a} d \underline{a}^{*}>{ }_{31} * b r \bar{a} d \underline{a}>_{69}$ nštok. brā.$d a$ (A sg. brāa $d u$ ) 'Bart' (D. Saraf). Vgl. Skok I 195 f., Snoj 2003: 53, Derksen 2008: 55.

brâv: ursl. *barwu $>{ }_{6} * b a r \beta u>{ }_{20} * b r \bar{a} \beta u>{ }_{25} * b r \bar{a} \beta b>{ }_{30} * b r \bar{a} \beta b>{ }_{39} * b r \bar{a} \beta b>{ }_{49}$ $* b r \underline{a} \beta>70$ nštok. $b r \underline{a} v$ (G sg. brāava) 'Hammel' (Šišić). Vgl. Skok I 202 f., Snoj 2003: 55, Derksen 2008: 58.

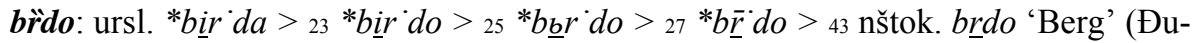
ričić). Vgl. Skok I 204 f., Snoj 2003: 56.

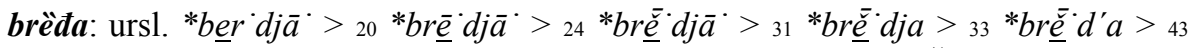
*bręd ${ }^{\prime} a>{ }_{60} * b r \underline{e} d^{\prime} a>71$ nštok. brẹđa 'trächtig (von Kühen)' (Šišić). Vgl. Trautmann 1970: 32, Skok I 206, Snoj 2003: 56, Derksen 2008: 36. 
brème: ursl. *ber $\dot{*} \bar{e} n>{ }_{20} * b r \underline{\bar{e}} \cdot m e \bar{e} n>{ }_{22} * b r \underline{\bar{e}} \cdot m e n>{ }_{24} * b r \underline{e} \cdot m e n>{ }_{28} * b r \underline{e} \cdot m \bar{e}>31$ *brê $m e ̨>{ }_{43}$ *brẹmę $>48$ *bręme $>60$ nštok. brẹme 'Last' (D. Saraf). Vgl. Trautmann 1970: 31 f., Skok I 201, Snoj 2003: 56, Derksen 2008: 37 („The accentuation of the etymon points to the presence of a laryngeal in the root.").

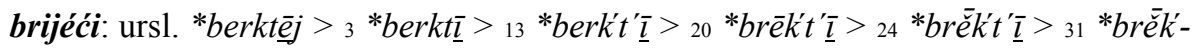

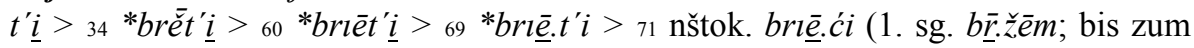
17. Jh. gebräuchlich, čakavisch noch heute) 'bewachen, bewahren, sorgen' (M. Saraf). Vgl. ARj I 647, Trautmann 1970: 31, Skok I 210, Derksen 2008: 36. Nach Dybo 1981: 208 konjugieren von den $e$-Verben mit Wurzeln auf Obstruent alle bis auf wenige von ihm genannte nach dem Akzentparadigma c.

brôd: ursl. *bradu 'Furt' $>23$ *brodu $>{ }_{25}$ *brodb $>{ }_{29} * b r \bar{o} d b>{ }_{30} * b r \underline{\bar{o}} d \boldsymbol{b}>{ }_{39} * b r \underline{\bar{\alpha}}-$ $d b>49$ nštok. brōd (G sg. bróda) 'Schiff' (D. Saraf). Vgl. Skok I 216, Snoj 2003: 60, Derksen 2008: 64.

br̂z: ursl. *burzu $>25 * b b r z b>{ }_{27} * b \bar{r} z b>{ }_{30} * b \underline{\bar{r}} z b>{ }_{39} * b \underline{\bar{r}} z b>49$ nštok. $b \underline{\bar{r}} z$ (fem. $b \underline{\bar{r}} . z a)$ 'schnell' (Berlakovich). Vgl. Skok I 222 f., Snoj 2003: 62, Derksen 2008: 70.

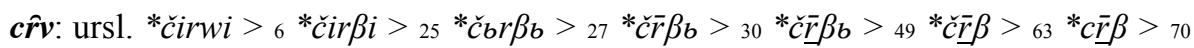
nštok. $c \bar{r} v$ 'Wurm' (Dobrić). Vgl. Skok I 276, Snoj 2003: 92, Derksen 2008: 93.

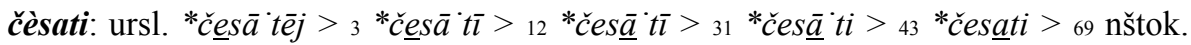
če.sati (1. sg. češsem) 'fricare, strigili mundare, pectere, vellere, carpere' (Đuričić). Vgl. ARj I 945, Skok I 311 f., Snoj 2003: 84, Derksen 2008: 86.

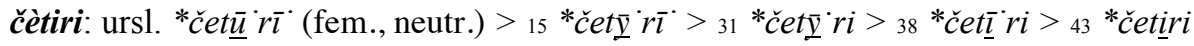
$>69$ nštok. če.tiri 'vier' (Đuričić). In Dialekten ist die maskuline Form auf -e (ursl. *četü 're) erhalten, siehe Jurišić 1973: 37. Vgl. noch Skok I 317 f., Snoj 2003: 741, Derksen 2008: 87.

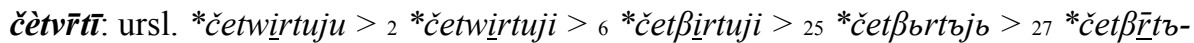

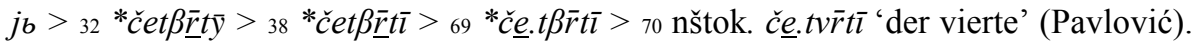
Siehe Jurišić 1973: 37 zu den dialektalen Akzentuierungen. Vgl. noch Skok I 317 f., Snoj 2003: 85 f., Derksen 2008: 87.

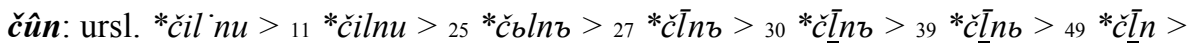
62 nštok. čûun, G sg. čūna 'Kahn' (Joachimsthal). Vgl. Skok I 342, Snoj 2003: 89.

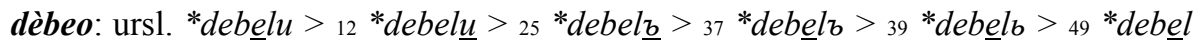
$>{ }_{64} *$ debeo $0>{ }_{69}$ nštok. de..beo (fem. debe. la) 'dick' (Pavlović). Vgl. Skok I 387, Snoj 2003: 97, Derksen 2008: $97 \mathrm{f}$.

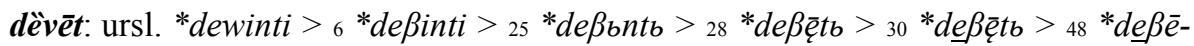
$t_{b}>{ }_{49} * d \underline{e} \beta \bar{e} t>70$ nštok. devēet 'neun' (Joachimsthal). Vgl. Skok I 398 f., Snoj 2003: 105 f., Derksen 2008: 101. 
dio: ursl. *dajlu $>{ }_{3} * d \underline{e} l u>{ }_{12} * d \bar{e} l \underline{u}>_{24} * d \bar{e} l \underline{u}>{ }_{25} * d \bar{E} l_{\underline{b}}>{ }_{37} * d \underline{\tilde{e}} \cdot l_{b}>{ }_{39} * d \underline{e} \cdot l_{b}>{ }_{49}$ $* d \underline{e} \cdot l>57 * d \underline{i} \cdot l>64$ nštok. dịo (G sg. dle e. $l a)$ 'Teil' (Berlakovich). Vgl. Skok I $406 \mathrm{f}$., Snoj 2003: 100, Derksen 2008: 103.

djềd: ursl. *dé $d u>{ }_{24} * d \underline{\bar{e}} d u>{ }_{25} * d \underline{\bar{e}} \cdot d b>{ }_{39} * d \underline{\bar{e}} \cdot d b>{ }_{43} * d \underline{\underline{e}} d b>{ }_{49} * d \underline{e} d>{ }_{60}$ *dıe $d>{ }_{66}$ nštok. djẹd 'Großvater' (Pavlović). Vgl. Skok I 411 f., Snoj 2003: 98, Derksen 2008: $101 \mathrm{f}$.

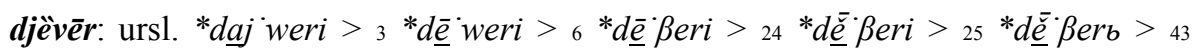

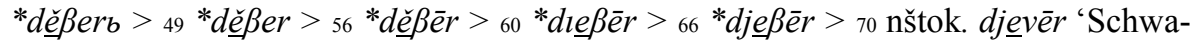
ger, Bruder des Manns' (Berlakovich). Vgl. Skok I 415, Jurišić 1973: 45, Dybo 1981: 18, Derksen 2008: 105.

dnö: ursl. *dinna 'Grund eines Gewässers' $>12 *$ din $\underline{a}>{ }_{23} *$ dino $\underline{{ }_{2}}>{ }_{25} *$ dbno $\underline{>}>49$ nštok. dno 'Boden' (Pavlović). Vgl. Skok I 418, Snoj 2003: 114. Im Akzentparadigma c wäre das auslautende - $o$ gelängt worden (s. Holzer 2007: $77 \S 49$ mit Fußnote $50)$.

dôm: ursl. *damu $>{ }_{23} *$ domu $>{ }_{25} *$ domъ $>29 *$ dōmъ $>{ }_{30} *$ dōmъ $>{ }_{39} *$ dōmb $>49$ nštok. dōm (G sg. dóma) 'Haus' (Berlakovich). Vgl. Skok I 424 f., Snoj 2003: 118, Derksen 2008: 113.

drâg: ursl. *dargu $>_{20} * d r a \bar{a} g u>{ }_{25} * d r \bar{a} g b>{ }_{30} * d r \bar{a} g b>{ }_{39} * d r \bar{a} g b>{ }_{49}$ nštok. $d r \underline{a} g$ (fem. $d r \underline{a} . g a)$ 'lieb, teuer' (Pavlović). Vgl. Skok I 428 f., Snoj 2003: 120, Derksen 2008: 113 .

dràga: ursl. *dar ${ }^{\prime} g \bar{a}{ }^{*}$ 'große Rinne im Gelände' $>{ }_{20} * d r \underline{a} \cdot g \bar{a} \cdot>_{31} * d r \underline{a} \cdot g a>{ }_{43}$ nštok. draga 'Tal, Fjord' (Pavlović). Vgl. Skok I 429, Snoj 2003: 120, Derksen 2008: 113 .

drága: ursl. * $\operatorname{darg} \underline{\bar{a}}{ }^{\prime}>{ }_{20} * d r \bar{a} g \underline{a}^{*}>{ }_{31} * d r \bar{a} g \underline{a}>_{69}$ nštok. $d r \underline{a} . g a$ 'lieb, teuer'. (Pavlović). Siehe $d r a ̂ g$.

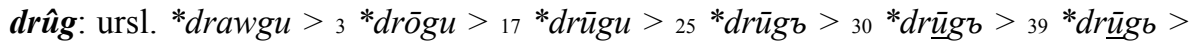
49 nštok. drūg (G sg. drūga) 'Gefährte' (Joachimsthal). Vgl. Skok I 446 f., Snoj 2003: 126 f., Derksen 2008: 121 f.

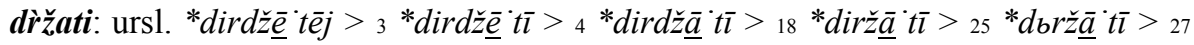

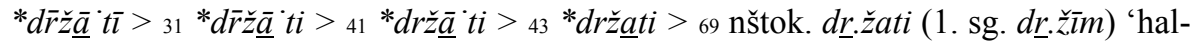
ten' (Joachimsthal). Vgl. Skok I 448 f., Snoj 2003: 127, Derksen 2008: 137 f.

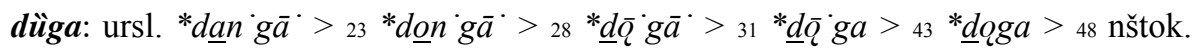
duga 'Daube' (Berlakovich).Vgl. Skok I 454, Snoj 2003: 115 f. 
dúga: ursl. *dang $\bar{a} \cdot{ }_{12} *$ dang $\underline{\bar{a}} \cdot{ }_{23} * \operatorname{dong}_{\underline{a}}{ }^{*}>{ }_{28} * \operatorname{dog} g \underline{\bar{a}}>_{31} * \operatorname{dog} g \underline{a}>{ }_{48} * \operatorname{du} g \underline{a}>$ 69 nštok. dū.ga (A sg. dū.gu) 'Regenbogen' (Berlakovich). Vgl. Skok I 453 f., Derksen 2008: 114.

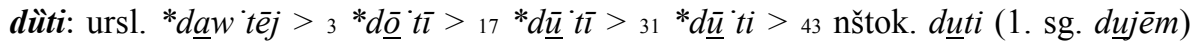
'wehen' (Joachimsthal). Vgl. ARj II 906 f., Skok I 462, Snoj 2003: 429 s. v. nadût.

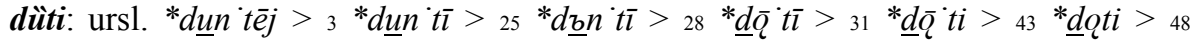
nštok. duti (1. sg. dmēm) 'blasen' (Joachimsthal). Vgl. ARj II 906 f., Skok I 462, Derksen 2008: $114 \mathrm{f}$.

glâd: ursl. *galdu $>20 *$ glādu $>25 *$ glādb $>30 *$ glāa $d b>{ }_{39} * g l \underline{a} d b>49$ nštok. glāa (G sg. glāada) 'Hunger' (Šišić). Vgl. ARj III 140, Skok I 563 f., Snoj 2003: 173, Derksen 2008: $173 \mathrm{f}$.

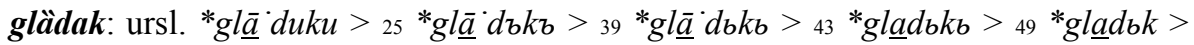
55 nštok. gladak 'glatt' (Joksimović). Vgl. Skok I 564, Snoj 2003: 173.

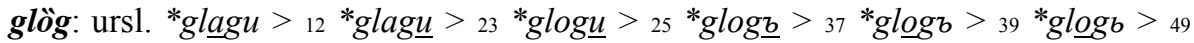
nštok. glog (G sg. glo.ga) 'Hagedorn' (Dobrić). Vgl. Skok I 571, Snoj 2003: 175, Derksen 2008: 166.

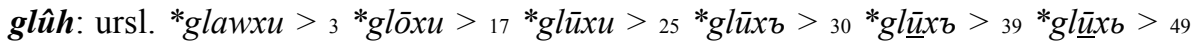

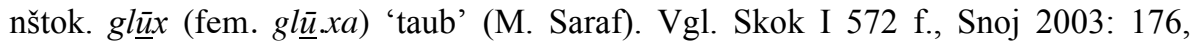
Derksen 2008: 167.

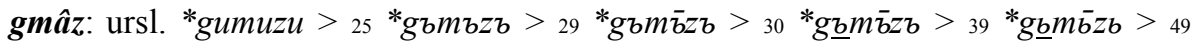

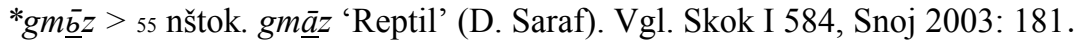

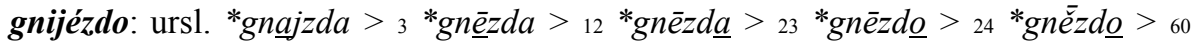
*gniēzdo > 69 nštok. gniê..zdo 'Nest' (Joksimović). Vgl. Skok I 576, Snoj 2003: 177, Derksen 2008: 169.

gòra: ursl. *garāa ${ }^{*}>{ }_{23}$ *gor $^{*}{ }^{*}>31$ *gora $>{ }_{69}$ nštok. go.ra (A sg. goru) 'Berg, Wald' (Joksimović). Vgl. Skok I 589-591, Vukušić 2007: 74 § 251, Snoj 2003: 182, Derksen 2008: $177 \mathrm{f}$.

gôst: ursl. *gasti $>23$ *gosti $>25{ }_{25}$ gostb $>29$ *gōstb $>30 *$ gōstb $>49$ nštok. gōost (G sg. gosta) 'Gast' (Joksimović). Vgl. Skok I 595 f., Snoj 2003: 184, Derksen 2008: $180 \mathrm{f}$.

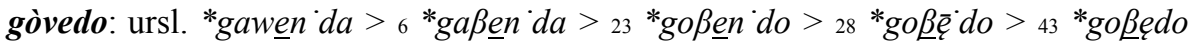

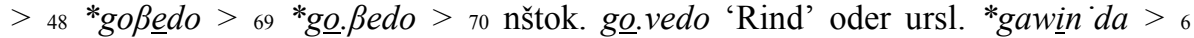

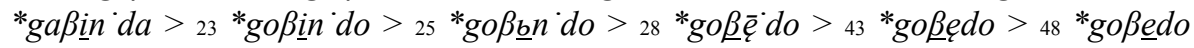
$>{ }_{69}$ *go. . 2003: 185, Derksen 2008: 181. 
gräd: ursl. $*_{g r \underline{a}} \cdot d u>{ }_{25} *_{g r \underline{a}} \cdot d b>{ }_{39} *_{g r \underline{a}} \cdot d b>{ }_{43} *_{g r \underline{a}} d b>49$ nštok. grad 'Hagel' (Joachimsthal). Vgl. Skok I 604, Derksen 2008: 185.

gräh: ursl. *gar $x u$ 'Erbse' $>{ }_{20} *_{g r \underline{a}} x u>{ }_{25} *_{g r \underline{a}} x_{b}>{ }_{39} *_{g r \underline{a}} x_{b}>{ }_{43} *_{g r \underline{a x b}}>49$ nštok. grax 'Bohne' (Joksimović). Vgl. Skok I 604 f., Snoj 2003: 187, Derksen 2008: 179 .

gröb: ursl. *grabu $>12 *_{\text {grabu }}>_{23} *_{\text {grob } \underline{u}}>25 *_{\text {grob } \underline{b}}>37 *_{\text {grobb }}>{ }_{39} *_{\text {grobb }}>49$ nštok. grob (G sg. gro.ba) 'Grab' (Pavlović). Vgl. ARj III 453, Skok I 601, Snoj 2003: 192, Derksen 2008: 190.

hôd: ursl. *xadu $>_{23} * x o d u>{ }_{25} * x o d b>{ }_{29} * x \bar{o} d b>{ }_{30} * x \underline{o} d b>{ }_{39} * x \bar{o} d b>49$ nštok. $x \underline{o} d$ 'Gang' (Dobrić). Vgl. Skok I 675, Snoj 2003: 207, Derksen 2008: 203.

igla: ursl. $*_{\text {igula }} \cdot{ }_{21} *_{j i g u l \underline{a}}>_{25} *_{j b g b l \underline{a}}>_{31} *_{j b g b l \underline{a}}>_{39} *_{j b g b l \underline{a}}>{ }_{40} *_{i g b l \underline{a}}>49$ *igla $>69$ nštok. i.gla (mit anscheinend sekundärem A sg. iglu) 'Nadel' (Dobrić). Vgl. ARj III 771, Skok I 710, Derksen 2008: 210.

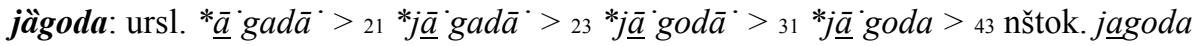
'Erdbeere' (Berlakovich). Vgl. Skok I 747, Snoj 2003: 233.

jälov: ursl. $*_{\underline{a}} \cdot l a w u>{ }_{6} * \underline{\bar{a}} \cdot l a \beta u>{ }_{21} * j \underline{\bar{a}} \cdot l a \beta u>{ }_{23} * j \underline{a} \cdot l o \beta u>{ }_{25} * j \underline{\bar{a}} \cdot l o \beta b>{ }_{39} * j \underline{a} \cdot l o-$ $\beta b>_{43} * j \underline{a}$ lo $\beta b>_{49} * j \underline{a} l o \beta>70$ nštok. jalov 'unfruchtbar' (Sivić). Vgl. Skok I 751 f., Snoj 2003: $233 \mathrm{f}$.

jäma: ursl. $*_{j} \underline{a} \cdot m \bar{a} \cdot>{ }_{2} *_{j} \underline{\underline{e}} \cdot m \bar{a} \cdot>_{4} *_{j} \underline{a} \cdot m \bar{a} \cdot>{ }_{31} * j \underline{a} \cdot m a>{ }_{43}$ nštok. jáama 'Grube' (Joksimović). Vgl. Skok I 752, Snoj 2003: 234, Derksen 2008: 28.

jâr: ursl. *jā'ru $>{ }_{2} * j \bar{e} \cdot r u$ (oder schon ursl. $\left.* j \bar{e} \cdot r u\right)>{ }_{4} * j \bar{a} \cdot r u>{ }_{11} * j \bar{a} r u>{ }_{25} * j \bar{a} r b>$ ${ }_{30} * j \underline{a} r b>39 * j \underline{a} r b>49$ nštok. $j \underline{a} r$ 'Frühjahr(s-)' (Dobrić). In etymologischem Zusammenhang mit jarac, s. dort. Vgl. ARj IV 463, Skok I 755 f., Snoj 2003: 234 f., Derksen 2008: 152 .

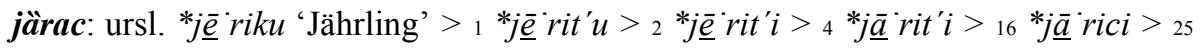
${ }^{*} \underline{j} \underline{a} \cdot r b c b>{ }_{43} *_{j} \underline{a r b c b}>{ }_{49} * j \underline{a r b c}>55$ nštok. jarac (G sg. jāarca, s. dort) 'Ziegenbock'

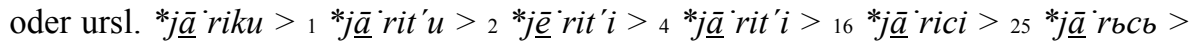
$43 *_{j} \underline{a r b c b}>{ }_{49} *_{j} \underline{a r b c}>55$ nštok. jarac (D. Saraf). In etymologischem Zusammenhang mit jāar , s. dort. Vgl. ARj IV 464, Skok I 755 f., Snoj 2003: 235, Derksen 2008: 153.

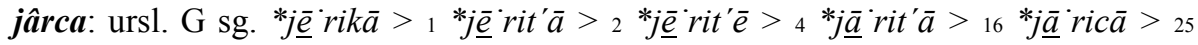

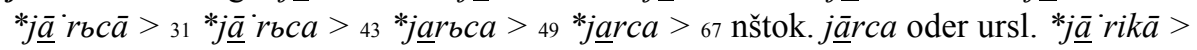

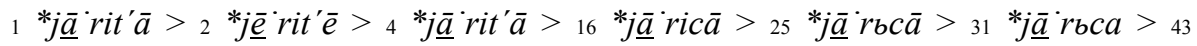
*jarbca $>{ }_{49} *_{j}$ jarca $>67$ nštok. jā $r c a$ (M. Saraf), siehe jarac. 
jästrēe: ursl. * $\underline{\bar{a}}$ strembu $>21 * j \underline{\bar{a}}$ strembu $>{ }_{25} * j \underline{\bar{a}}$ strembb $>28 * j \underline{\bar{a}}$ stręębr $>39$

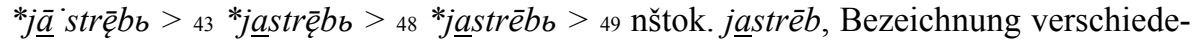
ner Raubvögel (Pavlović). Vgl. ARj IV 484 f., Skok I 760, Snoj 2003: 236, Derksen 2008: 29 .

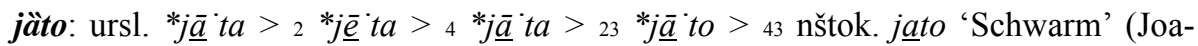
chimsthal). Vgl. ARj IV 489, Skok I 756 (s. v. jât), Snoj 2003: 236 (s. v. játa), Derksen 2008: 154.

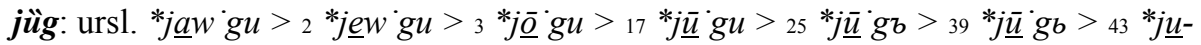
$g_{b}>49$ nštok. jug 'Süden' (Berlakovich). Vgl. Skok I 784 f., Snoj 2003: 244, Derksen 2008: $207 \mathrm{f}$.

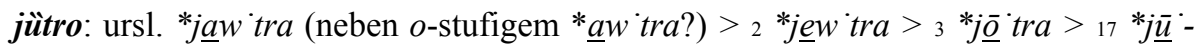
tra $>{ }_{23} *_{j} \underline{\underline{u}}$ 'tro $>43$ nštok. jütro 'Morgen' (Sivić). Vgl. Skok I 787 f., Snoj 2003: 245, Derksen 2008: $510 \mathrm{f}$.

káditi: ursl. $* k \bar{a} \cdot d \underline{\imath} \cdot t \bar{e} j>_{3} * k \bar{a} \cdot d \underline{\imath} \cdot t \bar{l}>{ }_{11} * k \bar{a} d \underline{\imath} \cdot t \bar{l}>{ }_{31} * k \bar{a} d \underline{i} \cdot t i>{ }_{43} * k \bar{a} d \underline{i} t i>{ }_{69}$ nštok.

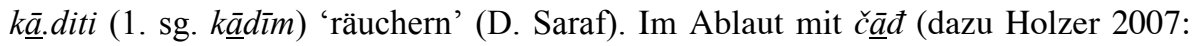
95; zur Rekonstruktion des Akuts s. ibidem und vgl. Derksen 2008: 77 s. v. *čàdjb). Vgl. ARj IV 730, Skok II 13, Snoj 2003: 247, Derksen 2008: 218 f. (auch zum heutigen Akzentparadigma b).

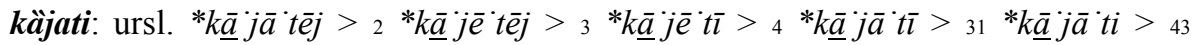
nštok. kajati (1. sg. kajēm) 'rächen; reflexiv: bereuen' (D. und M. Saraf). Vgl. ARj IV 737 f., Skok II 15 f.

kàlina: ursl. *kālej $n \bar{a} \cdot>_{3} * k \underline{a} l \bar{l} \cdot n \bar{a} \cdot>_{12} * k a \bar{a} l \underline{i} \cdot n \bar{a} \cdot>_{31} * k a \bar{a} l \underline{i} \cdot n a>{ }_{41} * k a l \underline{i} \cdot n a>43$ *kalinna $>69$ nštok. ka .lina (G pl. kạlīnā) 'Ligustrum vulgare L.' oder ursl. *kālej $n \bar{a}$ ' $>_{3} * k \bar{a} \underline{\imath}_{\bar{\imath}} n \bar{a} \cdot>{ }_{31} * k \bar{a} l \underline{i} \cdot n a>{ }_{41} * k a l \underline{i} \cdot n a>{ }_{43} * k a l \underline{i n} a>{ }_{69}$ nštok. ká.lina (Dobrić). Vgl. ARj IV 771, Skok II 21, Snoj 2003: 251.

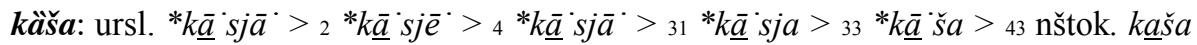
'Brei' (D. Saraf). Vgl. Skok II 59, Snoj 2003: 262.

kâuka: ursl. $* k \underline{\bar{a}} \cdot w u k \bar{a} \cdot>{ }_{6} * k \underline{\bar{a}} \cdot \beta u k \bar{a} \cdot>{ }_{25} * k \underline{\bar{a}} \cdot \beta b k \bar{a} \cdot>_{31} * k \underline{a} \cdot \beta b k a>{ }_{39} * k \underline{a} \cdot \beta b k a>$ ${ }_{43} * k \underline{a} \beta b k a>{ }_{49} * k \underline{a} \beta k a>{ }_{67} * k \underline{a} \beta k a>70$ nštok. $k \underline{a} v k a$ 'Corvus monedula L.' (M. Saraf). Vgl. ARj IV 907, Skok II 68, Snoj 2003: 266.

kìdati: ursl. ${ }^{*} k \underline{u} \cdot d \bar{a} \cdot t \overline{e j}>{ }_{3} * k \underline{u} \cdot d \bar{a} \cdot t \bar{l}>{ }_{15} * k \bar{y} \cdot d \bar{a} \cdot t \bar{l}>{ }_{31} * k \overline{\underline{y}} \cdot d \bar{a} \cdot t i>{ }_{38} * k \underline{\underline{u}} \cdot d \bar{a} \cdot t i>{ }_{43}$ nštok. kidati 'reißen' (Pavlović). Vgl. Skok II 77, Snoj 2003: 270, Derksen 2008: $264 \mathrm{f}$.

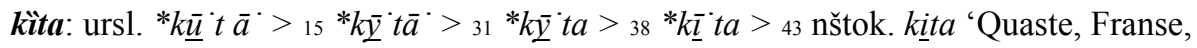
Strauß' (Joachimsthal). Vgl. Skok II 86, Snoj 2003: 273. 
kläda: ursl. $* k \underline{a l}{ } d \bar{a}^{*}>{ }_{20} * k l \underline{a} \cdot d \bar{a} \cdot>{ }_{31} * k l \underline{a} \cdot d a>{ }_{43}$ nštok. klạda 'Klotz' (Berlakovich). Vgl. Skok II 87, Snoj 2003: 273, Derksen 2008: 228.

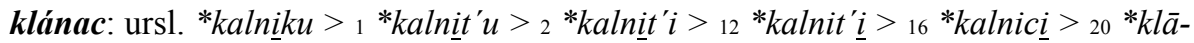
$n i c \underline{i}>{ }_{25} * k l \bar{a} n b c \underline{b}>{ }_{37} * k l a \bar{n} n \underline{b} c b>{ }_{49} * k l a \bar{n} \underline{\underline{b}} c>{ }_{55} * k l \bar{a} n \underline{a} c>{ }_{69}$ nštok. klā.nac (G sg. klā.nca) 'Engpass, Schlucht' (Sivić). Vgl. Skok II 88 f., Snoj 2003: 275.

klijêt: ursl. *klē $t i>{ }_{11} * k l e \overline{t i}>{ }_{24} * k l e \bar{t} t i>{ }_{25} * k l e \check{t} t b>{ }_{30} * k l \underline{e} t b>{ }_{49} * k l \underline{e} t>{ }_{60}$ nštok. $k l \underline{\underline{e}} t$ (fem., G sg. kliêti) 'bestimmte Art von Nebengebäude' (D. Saraf). Vgl. Skok II 99, Snoj 2003: 279, Derksen 2008: $224 \mathrm{f}$.

kljûč: ursl. *kljâwčju $>{ }_{2} * k l j \underline{e} w c ̌ j i>{ }_{3} * k l j \underline{o} c ̌ j i>{ }_{12} * k l j o ̄ c ̌ j \underline{j}>{ }_{17} * k l j \bar{u} c ̌ j \underline{i}>{ }_{25} * k l j \bar{u} c ̌ j \underline{b}$

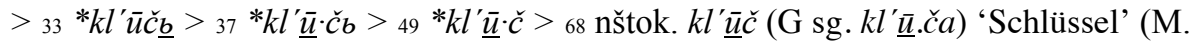
Saraf). Vgl. Skok II 104 f., Snoj 2003: 281, Derksen 2008: 226.

kljüka: ursl. ${ }^{*} k l j \underline{a} w{ }^{\cdot} k \bar{a}>_{2} * k l j \underline{e} w{ }^{*} k \bar{a} \cdot>_{3} * k l j \underline{a} \cdot k \bar{a} \cdot>_{17} * k l j \underline{u} \cdot k \bar{a} \cdot>_{31} * k l j \underline{u} \cdot k a>33$ $* k l^{\prime} \underline{u} \cdot k a>43$ nštok. $k l^{\prime} \underline{u} k a$ 'Haken' (Joksimović). Vgl. Skok II 105 (s. v. kljîǔ ), Derksen 2008: 226.

kòbila: ursl. ${ }^{*} k \underline{a} b \bar{u} \cdot l \bar{a} \cdot>_{12} * k a b \underline{\underline{u}} \cdot l \bar{a} \cdot($ oder schon ursl. $* k a b \underline{\underline{u}} \cdot l \bar{a} \cdot)>_{15} * k a b \bar{y} \cdot l \bar{a} \cdot>_{23}$

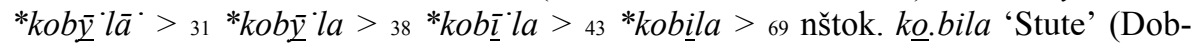
rić). Vgl. Skok II 143, Snoj 2003: 286.

kòsa: ursl. *kas $\underline{\bar{a}} \cdot>23$ *kos $\underline{\bar{a}} \cdot>31$ *kos $\underline{a}>69$ nštok. kㅁ.sa (A sg. kosu) 'Haar' (Đuričić). Im Ablaut zu če.sati. Vgl. Skok II 161, Derksen 2008: 238.

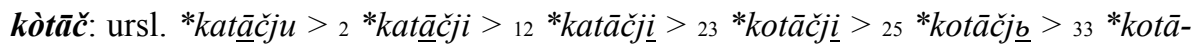
$\check{c} \underline{b}>{ }_{37} * k o t \bar{a} \cdot \check{c} b>{ }_{49} * k o t \underline{\bar{a}} \cdot \check{c}>{ }_{68} * k o t \underline{a} \underline{c}>_{69}$ nštok. ko.tāč (G sg. kotāa. $\left.\check{c} a\right)$ 'Rad' (Pavlović). Vgl. Skok II 169.

krátak: ursl. *kartuku $>{ }_{12} * k a r \underline{u} k u>_{20} * k r a \bar{t} \underline{u} k u>{ }_{25} * k r a \bar{t} \underline{z} k b>{ }_{39} * k r a \bar{a} \underline{b} k b>49$ *krāttb $k>{ }_{55} * k r a \overline{t a} \underline{k}>{ }_{69}$ nštok. krāa.tak (fem. sekundär krāa.tka) 'kurz' (Joachimsthal). Vgl. ARj V 476, Skok II 182 f., Dybo 1981: 106, Snoj 2003: 319, Jurišić 1973: 97 (auf Vrgada akzentuiert, als wäre es ursl. *kartuku), Derksen 2008: 236. Beachte auch Holzer 2010: 48 f. mit Fußnote 24.

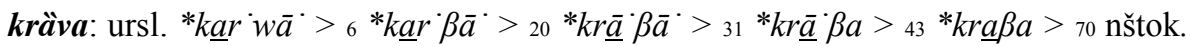
krava 'Kuh' (Berlakovich). Vgl. Skok II 183 f., Snoj 2003: 319, Derksen 2008: 236.

krílo: ursl. *krejdla $>{ }_{3} * k r \underline{i} d l a>{ }_{12} * k r \bar{u} d l \underline{a}>{ }_{19} * k r \bar{l} \underline{a}>{ }_{23} * k r i ̄ l \underline{o}>69$ nštok. $k r \underline{i} . l o$ 'Flügel' (Šišić). Vgl. Skok II 195 f., Snoj 2003: 324, Derksen 2008: 247.

kŕma: ursl. ${ }^{*} k \underline{u} r m \bar{a} \cdot{ }_{12} * k u r m \underline{a} \cdot{ }^{*}{ }_{25} * k b r m \underline{a} \cdot{ }_{27} * k \bar{r} m \underline{\bar{a}} \cdot>31 * k \bar{r} m \underline{a}>69$ nštok. $k \underline{\underline{r}} . m a$ (A sg. ${ }^{*} k \underline{\underline{r}} . m u$ ) 'Futter' (Sivić). Vgl. ARj V 597, Skok II 205 f., Snoj 2003: 326, Derksen 2008: 262. 


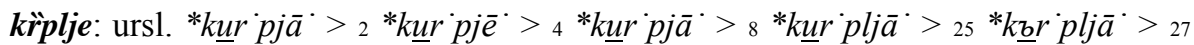
$* k \underline{r}{ }^{\prime} p l j \bar{a} \cdot>{ }_{31} * k \bar{r} \cdot p l j a>{ }_{33} * k \underline{r} \cdot{ }^{\prime} l^{\prime} a>43$ nštok. krpl'a, gebräuchlich im Plural krpl'e 'Schneeschuhe' (Joachimsthal). Vgl. ARj V 630, Skok II 211 (s. v. kr̀pa), Snoj 2003: 329 f., Derksen 2008: 263 (s. v. *kל̀rpa).

kr̈t: ursl. *krutu $>{ }_{12} * k r u t \underline{u}>25 * k r b t \underline{b}>27 * k r t \underline{b}>37 * k \underline{r} t b>39 * k \underline{r} t b>49$ nštok. $k \underline{r} t$ 'Maulwurf' (Joksimović). Vgl. ARj V 658, Skok II 213, Snoj 2003: 330.

krûg: ursl. *krangu $>23 * k r o n g u>{ }_{25} * k r o n g b>{ }_{28} * k r \bar{c} g b>30 * k \underline{r} \bar{g} g b>{ }_{39} * k \underline{r} \bar{q} g b$ $>{ }_{48} * k r \underline{u} g b>49$ nštok. krūg 'Kreis' (D. Saraf). Vgl. ARj V 661, Skok II 213 f., Snoj 2003: 327, Derksen 2008: 251.

krüh: ursl. *kraw $x u>_{3} * k r \underline{o} \cdot x u>{ }_{17} * k r \underline{\bar{u}} x u>{ }_{25} * k r \underline{u} \cdot x b>{ }_{39} * k r \underline{\bar{u}} x \boldsymbol{x}>{ }_{43} * k r \underline{u} x b$ $>49$ nštok. $k r \underline{u} x$ (G sg. kruxxa) 'Brot' (M. Saraf). Vgl. ARj V 663, Skok II 191, Snoj 2003: 331, Derksen 2008: 252.

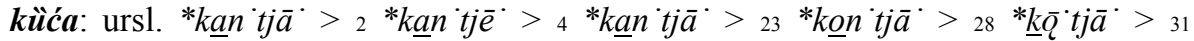
${ }^{*} \underline{k} \bar{c} \cdot t j a>{ }_{33} * \underline{k}_{\bar{c}} \cdot t^{\prime} a>{ }_{43}{ }^{*} \underline{k}_{\mathrm{c}} t^{\prime} a>{ }_{48} * k \underline{u} t^{\prime} a>71$ nštok. kúća 'Haus' (Dobrić). Vgl. ARj V 724, Skok II 221 f., Snoj 2003: 287, Derksen 2008: 244.

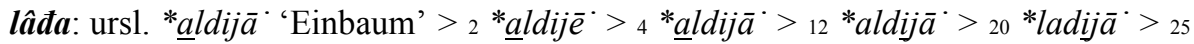

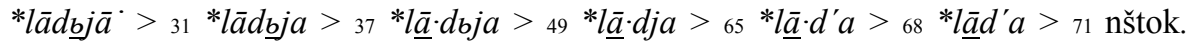
lāđa 'Schiff' (M. Saraf). Vgl. Skok II 259, Jurišić 1973: 103, Vasmer 1979: 52, Snoj 2003: 342, Derksen 2008: 367 (s. v. oldi).

làv: ursl. $* l_{\underline{i}} w u>{ }_{6} * l \underline{i} \beta u>{ }_{12} * l i \beta \underline{u}>{ }_{25} * l_{b} \beta \underline{\underline{b}}>{ }_{37} * l_{\underline{b}} \beta b>_{39} * l_{\underline{b}} \beta \underline{b}>{ }_{49} * l_{\underline{b}} \beta>{ }_{55} * l_{\underline{a}} \beta$

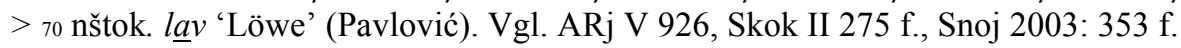

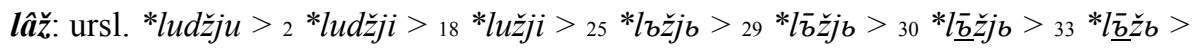
${ }_{39} * l_{\underline{b} z \check{z} b}>{ }_{49} * l_{\underline{b}} \underline{z}>{ }_{55}$ nštok. la $\underline{a} z \check{z}$ 'Lüge' (Joachimsthal). Vgl. Skok II 260 (s. v. làgati), Snoj 2003: 347, Derksen 2008: 295.

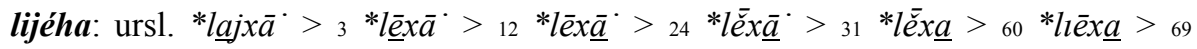
nštok. lie e.xa 'Beet' (Berlakovich). Vgl. Skok II 295 f., Snoj 2003: 350, Derksen 2008: 272.

lijên: ursl. *lē $n u>{ }_{11} * l e \bar{e} n u>{ }_{24} * l e \bar{n} n u>{ }_{25} * l \bar{e} n b>{ }_{30} * l \underline{\underline{e}} n b>{ }_{39} * l \underline{e} n b>49 * l \underline{e} n>60$ nštok. lıēn (fem. lie . $n a$, neutr. lıēno) 'faul' (Sivić). Vgl. Skok II 296, Snoj 2003: 351, Derksen 2008: 273.

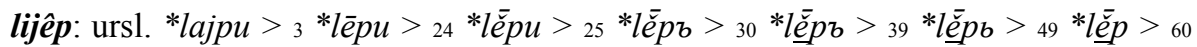

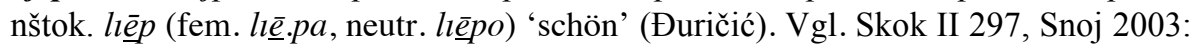
351 f., Derksen 2008: 274. 


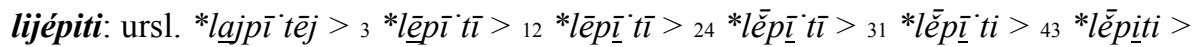

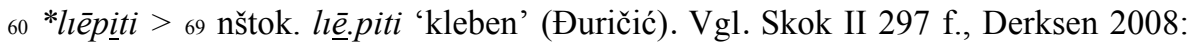
273.

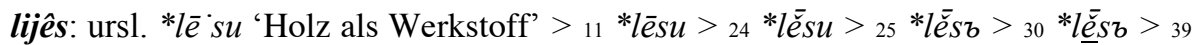

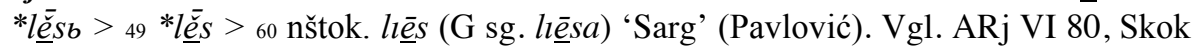
II 298, Derksen 2008: 274.

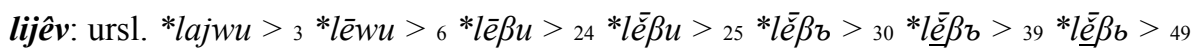

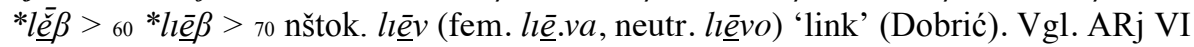
83, Skok II 299, Snoj 2003: 354 (rekonstruiert aber Akzentparadigma b), Jurišić 1973: 108 (entspricht Akzentparadigma c), Derksen 2008: 275.

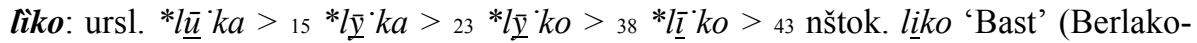
vich). Vgl. Skok II 301, Derksen 2008: 295 f.

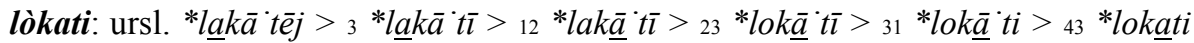
$>69$ nštok. lo.kati (1. sg. ločém) 'mit der Zunge trinken (von Hunden, Katzen u. dgl.)’ (Pavlović). Vgl. ARj VI 139, Skok II 314, Snoj 2003: 363, Derksen 2008: $283 \mathrm{f}$.

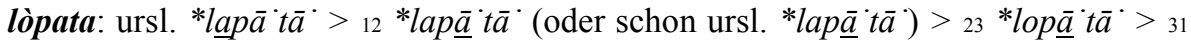

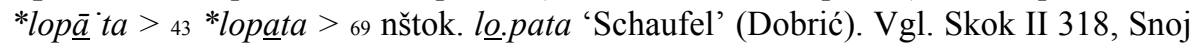
2003: 363, Kortlandt 1975: 16, Derksen 2008: 285.

lûb: ursl. *lawbu $>{ }_{3} * l \bar{o} b u>{ }_{17} * l \bar{u} b u>{ }_{25} * l \bar{u} b \boldsymbol{b}>{ }_{30} * l \underline{u} b b>39 * l \underline{u} b b>49$ nštok. $l \underline{u} b$ (G sg. lū $b a$ ) 'Baumrinde' (Joksimović). Vgl. Skok II 322 f., Snoj 2003: 366, Derksen 2008: 289.

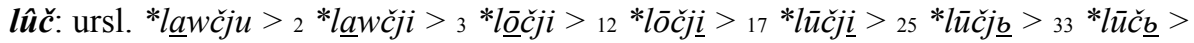
${ }_{37} * l \underline{\bar{u}} \cdot \check{c} b>{ }_{49} * l \underline{\bar{u}} \cdot \check{c}>68$ nštok. $l \underline{\bar{u}} \check{c}$ (G sg. $l \underline{\bar{u}} . \check{c} a$, daneben auch als femininer $i$-Stamm) 'Fackel, Kienspan' (D. Saraf). Vgl. Skok II 323 f., Snoj 2003: 367, Derksen 2008: 290.

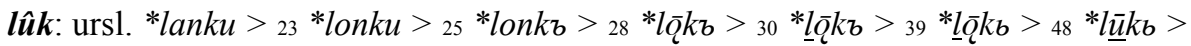

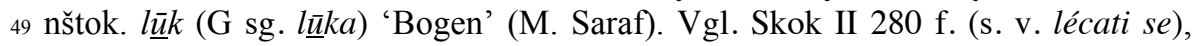
Snoj 2003: 362 f., Derksen 2008: 289.

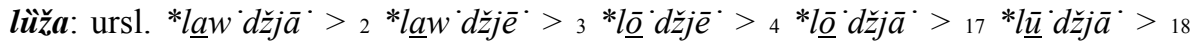

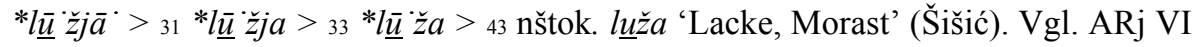
230, Skok II 333, Snoj 2003: 369, Derksen 2008: 293.

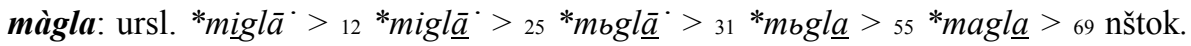
ma.gla (A sg. sekundär maglu) 'Nebel' (Berlakovich). Vgl. Skok II 353, Snoj 2003: 389, Derksen 2008: 338 f. (v. a. zur Akzentuierung). 


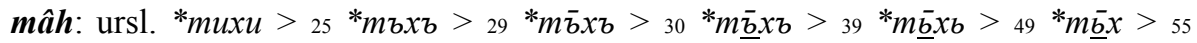
nštok. māx (G sg. maxa) 'muscus, Moos' (M. Saraf). Vgl. ARj VI 371, Skok II 354, Snoj 2003: 372 f., Derksen 2008: 333.

mäk: ursl. *māa $k u>{ }_{25} * m \underline{a} \cdot k b>{ }_{39} * m \underline{a} \cdot k b>{ }_{43} * m a k b b>49$ nštok. mak (G sg. maka neben sekundärem ma.ka, zu welchem s. Kapović 2006: 161) 'Mohn' (Šišić). Vgl. Skok II 358 f., Snoj 2003: 374, Derksen 2008: 299 f.

mäti: ursl. *māa $t \bar{l}>31{ }^{*} m \underline{a} \cdot t i>43$ nštok. mati 'Mutter' (Pičorušević). Vgl. Skok II 389, Snoj 2003: 385, Derksen 2008: 303.

mêd: ursl. *medu $>25 * m e d b>{ }_{29} * m \bar{e} d b>{ }_{30} * m \bar{e} d b>{ }_{39} * m \bar{e} d b>49$ nštok. $m \bar{e} d$ (G sg. méda) 'Honig' (Berlakovich). Vgl. Skok II 396 f., Snoj 2003: 387 f., Derksen 2008: $306 \mathrm{f}$.

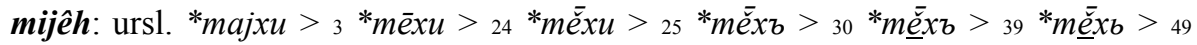

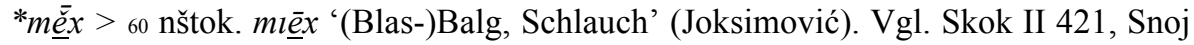
2003: 389, Derksen 2008: $309 \mathrm{f}$.

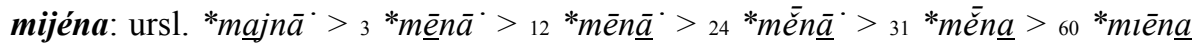
$>69$ nštok. mię.na (A sg. mię..nu) 'Wandel, Wechsel' (M. Saraf). Vgl. ARj VI 654, Skok II 421 f., Snoj 2003: 391, Derksen 2008: 311.

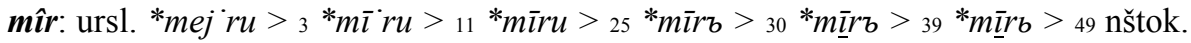
mīr 'Friede' (Pičorušević). Vgl. Skok II 426-428 (s. v. mïo), Snoj 2003: 403, Derksen 2008: 318. Die Akzentuierung des G sg. mī.ra (als wäre es ursl. **mejru gewesen) ist wohl sekundär. Bei Jurišić 1973: 119 erwartungsgemäß mī $r$ mī $r a$.

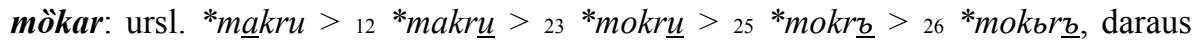
analogisch wie in ursl. *dabru > nštok. dobar (s. Holzer 2007: 97 s. v.) wieder *mo$k r \underline{b}>37 * m \underline{o} k r b>{ }_{39} * m \underline{o} k r b>46 * m \underline{o k b r b}>{ }_{49} * m \underline{o} k b r>55$ nštok. mokar (fem. sekundär mokra) 'nass' (Pičorušević). (Jurišić 1973: 122: mokar, fem. mokra , neutr. mokro.) Vgl. Skok II 449 f., Snoj 2003: 411, Derksen 2008: 322.

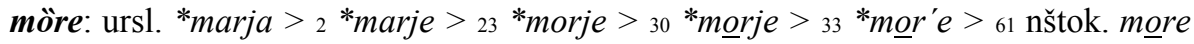
'Meer' (Pičorušević). Vgl. Skok II 455 f., Snoj Slovar 415, Derksen 2008: 325.

mrâk: ursl. *mar $k u>{ }_{11} *_{m a r k u}>20 * m r a ̄ k u>25 * m r a \overline{k b}>{ }_{30} * m r \underline{a} k b>39 * m r a \bar{k} k b$ $>49$ nštok. $m r \underline{a} a k$ (G sg. $m r \underline{a} k a$ ) 'Finsternis' (Šišić). Im Ablaut mit $m \underline{r} k$ (s. dort). Vgl. Skok II 468, Snoj 2003: 419, Derksen 2008: 325.

mràz: ursl. *mar $z u>{ }_{20} * m r \underline{a} \dot{z} z u>{ }_{25} * m r \underline{a} z z>{ }_{39} * m r \underline{a} \dot{a} z b>{ }_{43} * m r \underline{a} z b>49$ nštok. mraz (G sg. mraza) 'Frost' (Pičorušević). Im Ablaut mit mrznuti se (s. dort). Vgl. Skok II 472 f., Snoj 2003: 419, Derksen 2008: 326. 


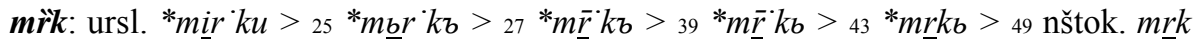
'dunkelbraun' (Joachimsthal). Im Ablaut mit mrāak (s. dort). Vgl. ARj VII 61, Skok II 467, Snoj 2003: 421, Derksen 2008: 342.

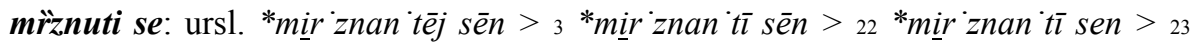

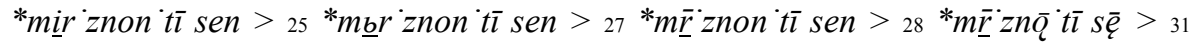

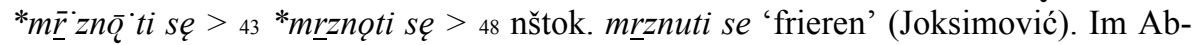
laut mit mraz (s. dort). Vgl. Skok II 472 f., Snoj 2003: 422, Derksen 2008: 342 f.

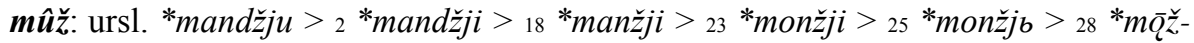

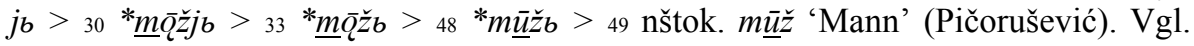
Skok II 492 f., Snoj 2003: 418, Derksen 2008: 330.

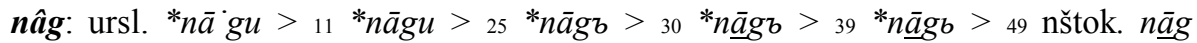
'nackt' (Joksimović). Vgl. Skok II 498, Snoj 2003: 429, Derksen 2008: 345.

nòga: ursl. *nag $\underline{\bar{a}} \cdot>23{ }^{*} n o g \underline{\bar{a}} \cdot>31 * n o g \underline{a}>69$ nštok. no.ga (A sg. nogu) 'Fuß' (Pavlović). Vgl. Skok II 522 f., Snoj 2003: 449, Derksen 2008: 354 f.

nòv: ursl. $*_{n \underline{a} w u}>{ }_{6} *_{n \underline{a} \beta u}>{ }_{12} *_{n a \beta \underline{u}}>{ }_{23} *_{n o} \beta \underline{u}>{ }_{25} *_{n o} \beta_{\underline{b}}>{ }_{37} *_{n \underline{o}} \beta b>{ }_{39} *_{n \underline{o}} \beta \boldsymbol{b}$

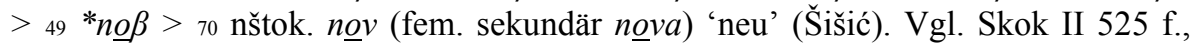
Snoj 2003: 451, Derksen 2008: 357. Lukežić - Zubčić 2007: 419: nôv, f. nova, n. novo (also sekundär nach Akzentparadigma c).

nôž: ursl: *nazju $>{ }_{2} *_{n \underline{a z j i}}>{ }_{12} *_{n a z j \underline{i}}>{ }_{23} *_{n o z j \underline{i}}>{ }_{25} *_{n o z j \underline{b}}>{ }_{33} *_{n o z ̌ b}>{ }_{37} *_{n \underline{z}} \underline{b}$ $>49$ nštok. $n \underline{\bar{o}} \check{z}$ (G sg. $n \underline{\bar{\alpha}} . \check{z} a)$ 'Messer' (Joachimsthal). Mit unerwarteter Länge. Vgl. Skok II 521 (s. v. nízati), Snoj 2003: 452, Derksen 2008: 358.

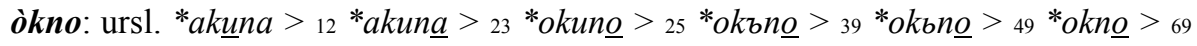
nštok. o.kno 'Fenster' (Pičorušević). Vgl. Skok II 551, Snoj 2003: 468, Derksen 2008: 365 .

ölovo: ursl. *alawa $>{ }_{6} * a l a \beta a>{ }_{23} *$ olo $\beta o>{ }_{30}{ }^{*} \underline{o l o} \beta o>70$ nštok. olovo 'Blei' (D. Saraf). Vgl. Skok II 554, Derksen 2008: 369 f.

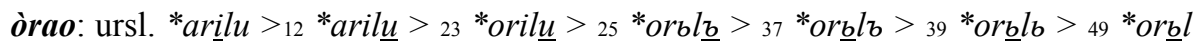
$>{ }_{55} *_{\text {oral }}>{ }_{64}$ *orao $>{ }_{69}$ nštok. o.rao (G sg. o.rla) 'Adler' (Šišić). Vgl. Skok II 563, Snoj 2003: 477, Derksen 2008: $376 \mathrm{f}$.

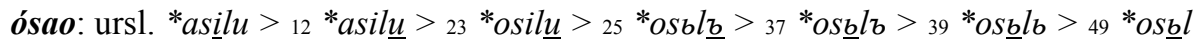
$>{ }_{55} *$ os $\underline{a l}>{ }_{64} *$ osao $>69$ nštok. o.sao (G sg. o.sla) 'Esel' (Đuričić). Vgl. Skok II 569, Snoj 2003: 479, Derksen 2008: 381.

óvna: ursl. G sg. *awinnā $>{ }_{6} * a \beta \underline{i n} n \bar{a}>{ }_{12} * a \beta i n \underline{\bar{a}}>{ }_{23} * o \beta i n \underline{\bar{a}}>{ }_{25} *$ o $\beta b n \underline{\bar{a}}>{ }_{31} * o \beta b n \underline{a}$ $>{ }_{49} * o \beta n \underline{a}>{ }_{67} * \bar{o} \beta n \underline{a}>{ }_{69} * \underline{o} . \beta n a>70$ nštok. $\underline{0}$.vna $(\mathrm{N}$ sg. o.van) 'Hammel' (M. Saraf). Vgl. Skok III 579, Snoj 2003: 483, Derksen 2008: 384. 
óvsa: ursl. G sg. *awisis $>{ }_{6} * a \beta \underline{i} s \bar{a}>{ }_{12} * a \beta i s \underline{\bar{a}}>{ }_{23} * o \beta i s \underline{\bar{a}}>{ }_{25} * o \beta b s \underline{\bar{a}}>{ }_{49} * o \beta s \underline{\bar{a}}>$ ${ }_{67}^{*} \bar{o} \beta s \underline{\bar{a}}>{ }_{69} * \overline{\bar{o}} . \beta s a>70$ nštok. $\underline{\bar{o}} . v s a(\mathrm{~N}$ sg. $\underline{\text { o.vas}}$ ) 'Hafer' (D. Saraf). Vgl. ARj IX 482, Skok III 580, Snoj 2003: 483, Derksen 2008: 384 f.

päsmo: ursl. *pāa $s m a>{ }_{23} * p \underline{\bar{a}}$ 'smo $>43$ nštok. pasmo 'Strähne, Fitze (Weberterminus)' (Šišić). Vgl. Skok II 613, Snoj 2003: 496.

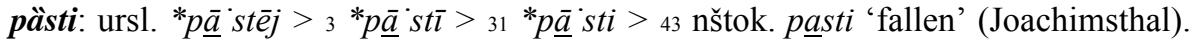
Vgl. Skok II 615 f., Snoj 2003: 497, Derksen 2008: 392.

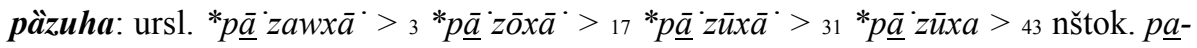
zuxa 'Achselhöhle' (Joachimsthal). Vgl. ARj IX 726, Skok II 662, Snoj 2003: 500 s. v. pâzduha, Derksen 2008: 392 s. v. *pazduxa.

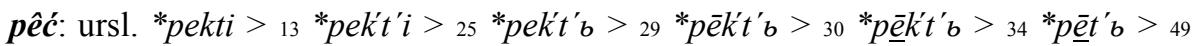

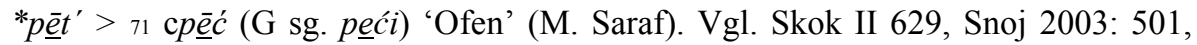
Derksen 2008: 393.

pëpeo: ursl. *pepelu $>25 *$ pepelb $>29 *$ pepēlb $>30 *$ pepēlb $>39 *$ pepélb $>49$ *pepēl $>64$ nštok. pepeo (G sg. pepela) 'Asche' (Pavlović). Vgl. Skok II 638, Snoj 2003: 505 f., Derksen 2008: 395.

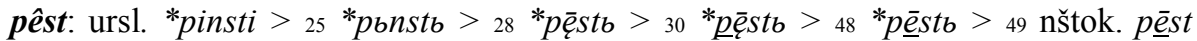
fem. (G sg. pēesti) 'Faust' (Joachimsthal). Schwundstufe zu pēet. Vgl. ARj IX 802, Skok II 645, Snoj 2003: 508, Derksen 2008: 399.

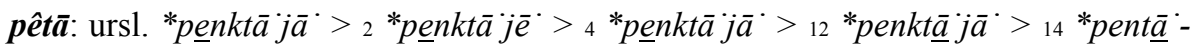

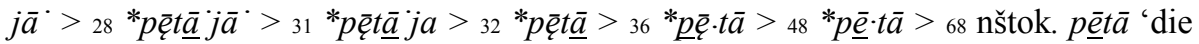
fünfte’ (Dobrić). Vgl. Skok II 647 f., Snoj 2003: 509 f., Derksen 2008: 399 f.

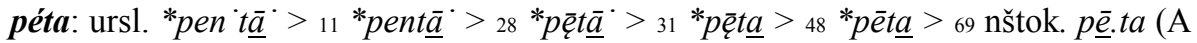
sg. pêetu) 'Ferse' (Dobrić). Vgl. Skok II 648, Snoj 2003: 510, Derksen 2008: 399.

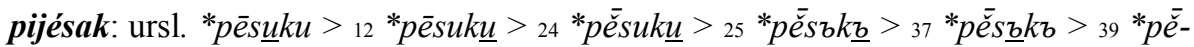
$s \underline{b} k b>{ }_{49} * p \bar{e} s \underline{b} k>{ }_{55} * p \check{e} s \underline{a} k>{ }_{60} * p i \bar{e} s \underline{a} k>69$ nštok. piēe.sak (G sg.piēe.ska) 'Sand' (Dobrić). Vgl. Skok II 654 f., Snoj 2003: 508, Derksen 2008: 398.

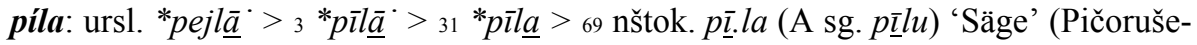
vić). Vgl. Skok II 656, Snoj 2003: 514.

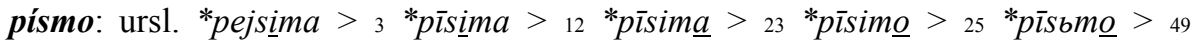
*pīsmo $>{ }_{69}$ pī.smo 'Schrift' (Joksimović). Vgl. Skok II 663, Snoj 2003: 516 f. (s. v. pisáti). 
plâv: ursl. ${ }^{*} p \underline{a} l w u>{ }_{6}{ }^{*} p \underline{a} l \beta u>{ }_{12} * p a l \beta \underline{u}>{ }_{20} * p l \bar{a} \beta \underline{u}>{ }_{25} * p l \bar{a} \beta \underline{b}>{ }_{37} * p l \underline{a} \cdot \beta b>39$ ${ }^{*}$ plā $\beta \beta b>{ }_{49} * p l \underline{\bar{a}} \cdot \beta>{ }_{68} * p l \underline{a} \beta>70$ nštok. plāa $v$ 'blond' (M. Saraf). Vgl. Skok II 679 f., Snoj 2003: 523 f.

prâh: ursl. *parxu $>{ }_{20} *$ prāxu $>{ }_{25} *$ prāx $b>{ }_{30} *$ prā $x$ z $>{ }_{39} *$ prāa $x \boldsymbol{b}>{ }_{49}$ nštok. prāa $x$ (G sg. prāxxa) 'Staub, Pulver' (Šišić). Vgl. Skok III 21 f., Snoj 2003: 557, Derksen 2008: 413.

präm: ursl. *par $m u>{ }_{20} * p r \underline{a} \cdot m u>{ }_{25} * p r \underline{a} \cdot m b>{ }_{39} * p r \underline{a} \cdot m b>{ }_{43} * p r \underline{a m b}>49$ nštok. pram 'Schiff’ (Dobrić). Vgl. ARj XI 361, Skok III 22.

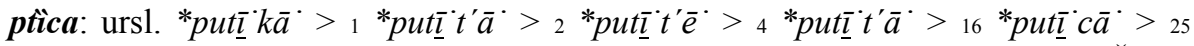

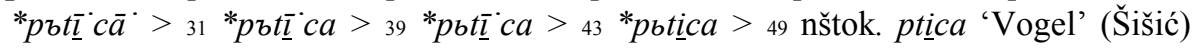

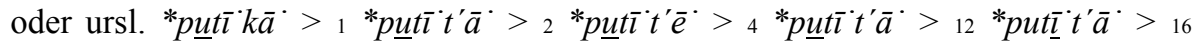

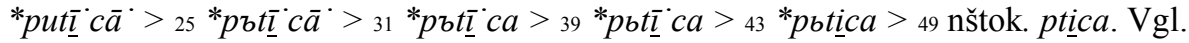
Skok III 64, Snoj 2003: 591, Derksen 2008: 425.

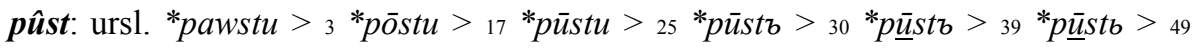
nštok. pū $s t$ 'leer, öde' (Berlakovich). Vgl. Skok III 82 f., Snoj 2003: 594, Derksen 2008: 424.

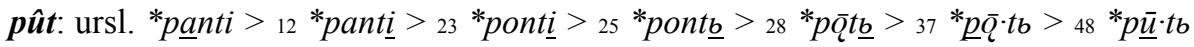
$>{ }_{49} * p \underline{\bar{u}} \cdot t>{ }_{68}$ nštok. $p \underline{u} t$ mask. (heute $o$-Stamm) 'Weg' (Pavlović). Vgl. Skok III 86 f., Snoj 2003: 552, Derksen 2008: 417 f.

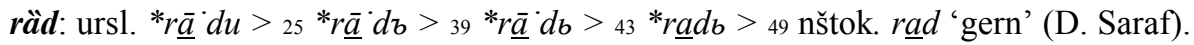
Vgl. Skok III 94 f., Snoj 2003: 598.

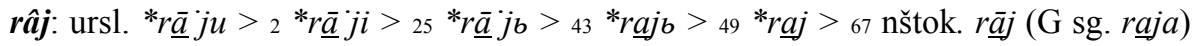
'Paradies' (D. Saraf). Vgl. Skok III 100, Snoj 2003: 600.

ràme: ursl. *ar $m \bar{e} n>10 * r \underline{a} \cdot m \bar{e} n>22 * r \underline{a} \cdot m e n>{ }_{28} * r \underline{a} \cdot m \bar{e}>{ }_{31} * r \underline{a} \cdot m e \underline{c}>43$ *rameq $>48$ nštok. rame 'Schulter' (Dobrić). Vgl. Skok III 105 f., Snoj 2003: 601, Derksen 2008: 375.

rân: ursl. *rānu $>{ }_{25} * r \bar{a} n b>{ }_{30} * r \underline{a} n b>{ }_{39} * r \underline{a} n b>49$ nštok. $r \underline{\bar{a}} n$ (Adv. ranon) 'früh' (Sivić). Vgl. Skok III 106, Snoj 2003: 601, Jurišić 1973: 177.

räna: ursl. ${ }^{*} \underline{\underline{a}} \cdot n \bar{a} \cdot>{ }_{31}{ }^{*} r \underline{\bar{a}} \cdot n a>43$ nštok. rana 'Wunde' (Pičorušević). Vgl. Skok III 107, Snoj 2003: $601 \mathrm{f}$.

râž: ursl. *rudži $>{ }_{18} *_{r u z ̌ i}>{ }_{25} *_{r b \check{z} b}>{ }_{29} *_{r \bar{b}} \check{z} b>{ }_{30} *_{r} \underline{\bar{z}} \check{z} b>{ }_{39} *_{r \underline{b}} \check{z} b>{ }_{49} *_{r \underline{b}} \check{z}>55$ nštok. $r \underline{a} \underline{z}$ (fem.; alter G sg. $\underline{r} \check{z} i$ ) 'Roggen' (Pičorušević). Vgl. Skok III 116, Snoj 2003: 635 . 
rèbro: ursl. *rébra $>12$ *rebra $>{ }_{23} *^{*} r e b r \underline{o}>69$ nštok. re $\underline{e}$ bro 'Rippe' (D. Saraf). Vgl. Skok III 119, Snoj 2003: 608, Derksen 2008: 433 und zu solchen Neutra Holzer 2005: 41 f. $§ 0.2$.

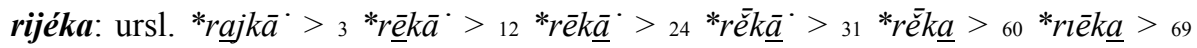
nštok. rlę..ka 'Fluss' (Berlakovich). Vgl. Skok III 141, Snoj 2003: 613, Derksen 2008: $434 \mathrm{f}$.

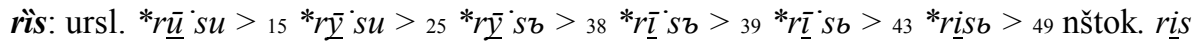
'Luchs' (Šišić). Vgl. Skok III 146, Snoj 2003: 623.

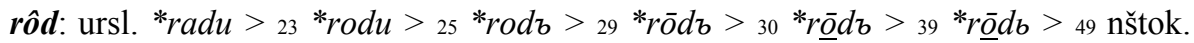
rōod (G sg. róda) 'Geschlecht' (D. Saraf). Vgl. Skok III 151-153, Snoj 2003: 626 f., Derksen 2008: 437.

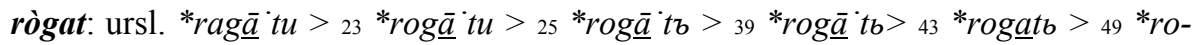
gát $>69$ nštok. ro. gat 'gehörnt' (D. Saraf). Vgl. lit. ragúotas, lett. raguôts. Vgl. Skok III 153 f., Trautmann 1970: 235, Holzer 2010: 67. Zu ursl. *ragu > rōg, zu welchem s. Holzer 2005: 40 f. § 0.1, 2007: 129.

ròsa: ursl. ${ }^{*}$ ras $\underline{\bar{a}}{ }^{\circ}>{ }_{25}{ }^{*}$ ros $\underline{\bar{a}} \cdot>{ }_{31}{ }^{*}$ ros $\underline{a}>69$ nštok. ro.sa (A sg. rosu) 'Tau' (M. Saraf). Vgl. Skok III 158 f., Snoj 2003: 630, Derksen 2008: 438 f.

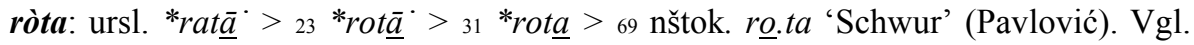
ARj XIV 181, Skok III 160 (s. v. rot), Snoj 2003: 630 (s. v. rotīti). Vgl. ròtiti.

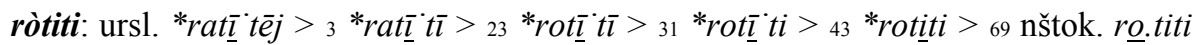
(1. sg. ro.tìm), hauptsächlich reflexiv 'sich verschwören' (Joachimsthal). Vgl. ARj XIV 185 f., Skok III 160 (s. v. rot), Snoj 2003: 630. Vgl. ròta.

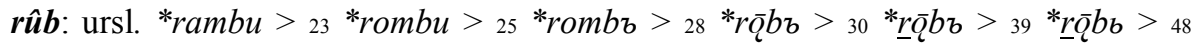
${ }^{*} r \underline{\bar{u}} b b>49$ nštok. $r \underline{\bar{u}} b$ (G sg. $\left.r \underline{\bar{u}} b a\right)$ 'Saum' (D. Saraf). Vgl. Skok III 163, Snoj 2003: 625.

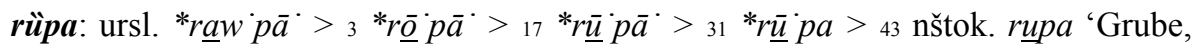
Loch' (Dobrić). Vgl. Skok III 172 f., Trautmann 1970: 240.

sâd: ursl. $*_{s \bar{a}} \cdot d u>{ }_{11} *_{s} \bar{a} d u>{ }_{25} *_{s \bar{a}} d \vec{b}>{ }_{30} *_{s \underline{a}} d b>{ }_{39} *_{s \underline{a}} d b>{ }_{49}$ nštok. $s \underline{\bar{a}} d$ (G sg. $s \underline{\bar{a}} d a$ ) 'Pflanzung' (Joachimsthal). Vgl. Skok III 184, Snoj 2003: 636, Derksen 2008: $442 \mathrm{f}$.

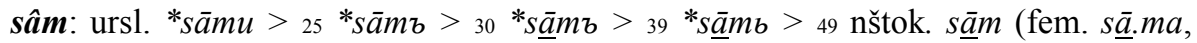
neutr. sa a.mo, in der Posavina und in Dubrovnik sāmo) 'selbst' (Dobrić). Vgl. ARj XIV 545, Skok III 197 f., Snoj 20o3: 639, Jurišić 1973: 97. 
sèdlo: ursl. *sedula $>{ }_{12} *_{\text {sedula }}>{ }_{23} *_{\text {sedulo }}>{ }_{25} *_{\text {sedblo }}>{ }_{39} *_{\text {sedblo }}>{ }_{49} *_{\text {sedlo }}>$ 69 nštok. se. .dlo (NA pl. sedla, G pl. sedā.lā) 'Sattel' (Pavlović). Vgl. ARj XIV 791, Skok III 213, Snoj 2003: 644, Derksen 2008: 443 f.

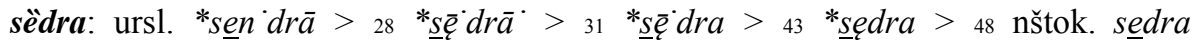
'Kalksinter' (Joksimović). Vgl. ARj XIV 799, Trautmann 1970: 256, Skok III 185 (s. v. sadra).

sèstra: ursl. ${ }^{*}$ sestra ${ }^{*}>{ }_{12} *_{\text {sestra }}{ }^{*}>{ }_{31} *_{\text {sestra }}>69$ nštok. se $\underline{\text { estra }}$ (V sg. sestro, G pl. sestā.rā) 'Schwester' (Joksimović). Vgl. ARj XIV 870, Skok III 226, Snoj 2993: 651, Derksen 2008: 445.

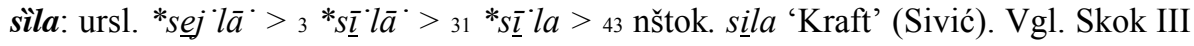
234 f., Snoj 2003: 654, Derksen 2008: 451.

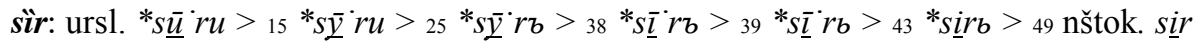
'Käse' (D. Saraf). Vgl. Skok III 242, Snoj 2003: 657, Derksen 2008: 483 f.

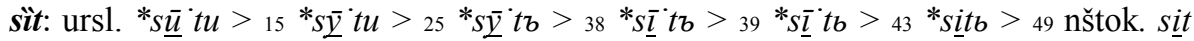
'satt' (Berlakovich). Vgl. Skok III 245, Snoj 2003: 658, Derksen 2008: 484.

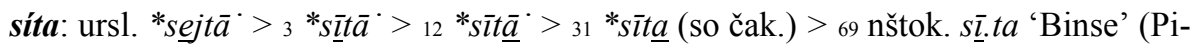
čorušević). Vgl. ARj XV 75, Trautmann 1970: 253, Skok III 245 f., Jurišić 1973: 190.

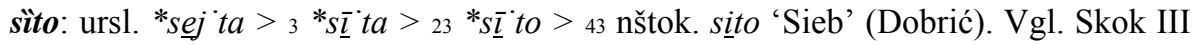
246, Snoj 2003: 658 f., Derksen 2008: 451.

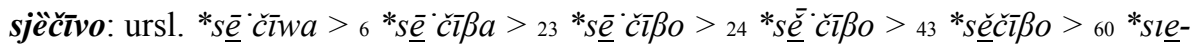

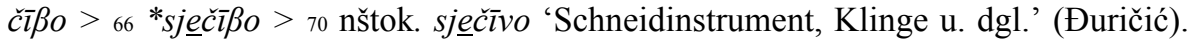
Vgl. ARj XV 105, Trautmann 1970: 255, Skok III 249. Zur zugrunde liegenden Verbalwurzel s. Dybo 1981: 209.

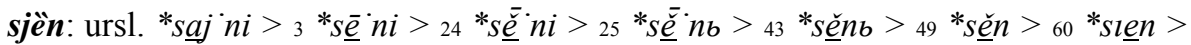
66 nštok. sjẹn mask. fem. (neben sjenna) 'Schatten' (M. Saraf). Vgl. ARj XV 148 f., Skok III 250 f., Snoj 2003: 648 (s. v. senca), Derksen 2008: 447.

skôk : ursl. *skaku $>_{23} *_{s k o k u}>_{25} *_{s k o k b}>_{29} *_{s k o ̄ k b}>{ }_{30} *_{s k \underline{o} k b}>{ }_{39} *_{s k \bar{o} k b}>{ }_{49}$ nštok. skōk (G sg. skoka; neben regionalem skok, G sg. skoka) 'Sprung' (Dobrić). Vgl. ARj XV 261, Skok III 263 f., Snoj 2003: 662 (rekonstruiert allerdings Akzentparadigma $b)$.

släb: ursl. *slāa $b u>{ }_{25} *_{s l \underline{\bar{a}} \cdot b b}>_{39} *_{s} l \underline{\bar{a}} \cdot b b>{ }_{43} *_{s l \underline{a}} b b>49$ nštok. sláb 'schwach' (D. Saraf). Vgl. Skok III 277, Snoj 2003: 665 f., Derksen 2008: 452. 


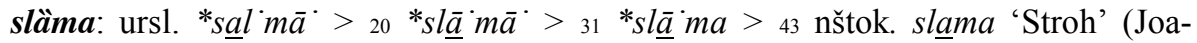
chimsthal). Vgl. Skok III 278 f., Snoj 2003: 666, Derksen 2008: 459.

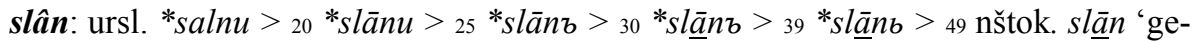
salzen' (M. Saraf). Zu sō, s. dort. Vgl. Skok III 303, Snoj 2003: 666, Derksen 2008 : 460 .

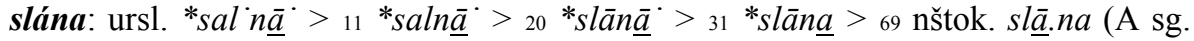
slānu) 'Reif' (Pavlović). Vgl. ARj XV 424, Skok III 303, Snoj 2003: 666 f., Derksen 2008: 460.

slâst: ursl. *salsti fem. $>20$ *slāsti $>25$ *slāstb $>30$ *slāstb $>49$ nštok. slāst fem. 'Genuss' (Šišić). Vgl. Skok III 278, Snoj 2003: 667.

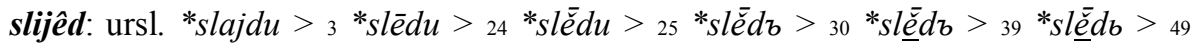

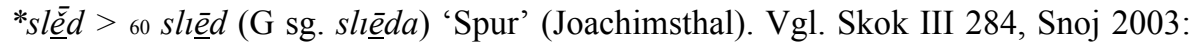
668.

slijêp: ursl. *slajpu $>_{3} *_{\text {slēpu }}>{ }_{24} *_{\text {slëpu }}>_{25} *_{\text {slëpb }}>{ }_{30} *_{\text {slëpb }}>{ }_{39} *_{\text {slëpb }}>49$

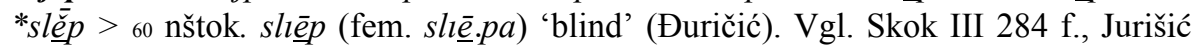
1973: 192, Snoj 2003: 668.

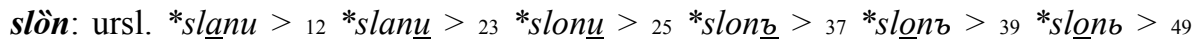
nštok. slon 'Elefant' (M. Saraf). Vgl. Skok III 286 f., Snoj 2003: 670.

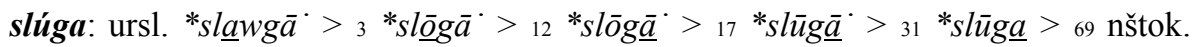
slü.ga 'Diener' (Pavlović). Vgl. ARj XV 613 f., Skok III 287, Snoj 2003: 672.

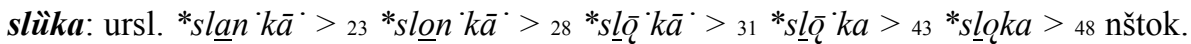
slukka 'ficedula, Schnepfe' (M. Saraf). Im Baltischen ohne Akut: lit. slankà, A sg. slañka 'Schnepfe', lett. sluõka 'Waldschnepfe'. Vgl. ARj XV 622, Trautmann 1970: 268, Skok III 405 (s. v. šjlüka), Snoj 2003: 670, Derksen 2008: 454.

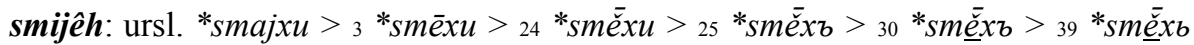

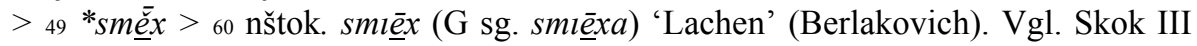
292, Snoj 2003: 673, Derksen 2008: 456.

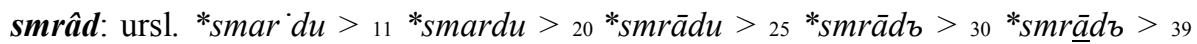
${ }^{*} s m r \underline{a} d b>49$ nštok. smrāad 'Gestank' (Sivić); o-Stufe zu smri. djeti (s. dort). Vgl. Skok III 295, Snoj 2003: 675, Derksen 2008: 456.

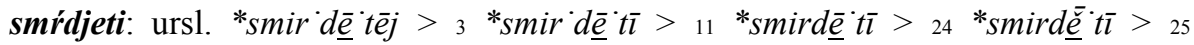

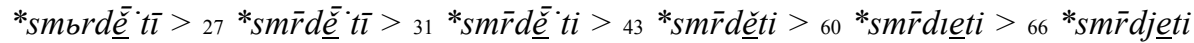

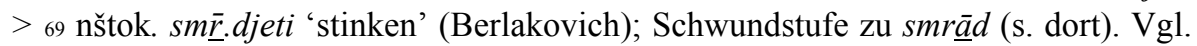
Skok III 294 f., Snoj 2003: 675, Derksen 2008: 457. 


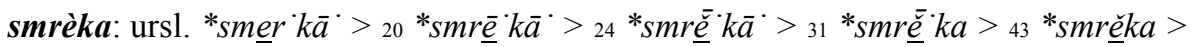
60 nštok. smreka 'Fichte' (D. Saraf). Vgl. Skok III 295, Snoj 2003: 675

snijêg: ursl. *snajgu $>_{3} *_{s n e ̄ g u}>_{24} *_{\text {snĕgu }}>{ }_{25} *_{\text {sněgz }}>{ }_{30} *_{\text {snĕg } b}>{ }_{39} *_{\text {snĕgb }}>$ 49 *snēg > 60 nštok. sniēg 'Schnee' (Sivić). Vgl. ARj XV 844, Skok III 297, Snoj 2003: 676 f., Derksen 2008: 457.

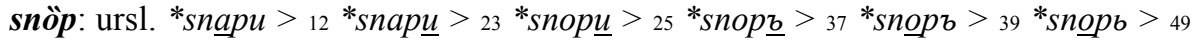
snop (G sg. sno.pa) 'Garbe' (Joksimović). Vgl. Skok III 298, Snoj 2003677 und snōpl'e in Holzer 2007: $131 \mathrm{f}$.

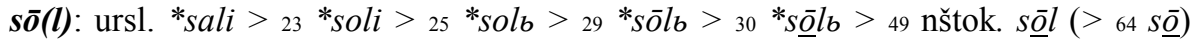
'Salz' (Đuričić). Davon slānn 'gesalzen', s. dort. Vgl. Skok III 303 f., Snoj 2003: 680, Derksen 2008: 461 und Holzer 2007: $84 \S 64$ zur stilistischen Frage - $\bar{o}:-\bar{o} l$.

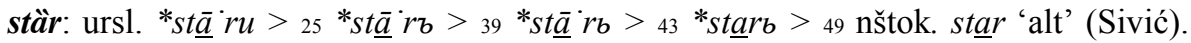
Vgl. Skok III 328, Snoj 2003: 694, Derksen 2008: 465.

stöbōr: ursl. *stabaru $>{ }_{23} *_{\text {stoboru }}>{ }_{25} *_{\text {stoborb }}>{ }_{29} *_{\text {stobōrb }}>{ }_{30} *_{\text {stobōrb }}>39$ $*_{\text {stóbōrb }>} 49$ nštok. stọbōr 'Gehege, Hof, Hofummauerung' (Pavlović). Vgl. ARj XVI 596, Skok III 336 f.

stôg: ursl. *stagu $>{ }_{12} *_{\text {stag } \underline{u}}>{ }_{23} *_{\text {stog } \underline{u}}>{ }_{25} *_{\text {stog }} \underline{\underline{b}}>{ }_{37} *_{\text {stog }}>{ }_{39} *_{\text {stog }}>{ }_{49}$ nštok. stōg (mit unerwarteter Länge; G sg. sto.ga) 'Schober' (Pičorušević). Vgl. ARj XVI 598, Skok III 337, Snoj 2003: 700, Derksen 2008: 468.

stöžēr: ursl. *stadžeru $>{ }_{18} *_{\text {stažeru }}>{ }_{23} *_{\text {stožeru }}>{ }_{25} *_{\text {stožerb }}>{ }_{29} *_{\text {stožērb }}>30$ *stožērb > 39 *stožērb > 49 nštok. stožēer 'Dreschpflock, Türangel' (Berlakovich). Vgl. ARj XVI 636, Trautmann 1970: 285, Skok III 337, Snoj 2003: 702.

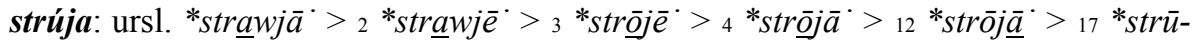
$j \underline{\bar{a}} \cdot{ }_{31} *_{s t r u \bar{j} j \underline{a}}>69$ nštok. str $\underline{u}$.ja 'Strömung' (Joachimsthal). Vgl. Skok III 349 f., Derksen 2008: 470.

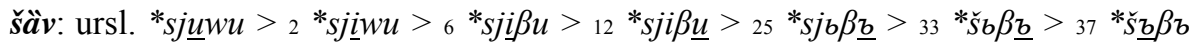
$>{ }_{39} *_{\breve{s}} \underline{\beta} \beta b>{ }_{49} *_{\breve{s}} \underline{\beta} \beta>{ }_{55} *_{\check{s}} \underline{a} \beta>70$ nštok. $\check{s} \underline{a} v$ (G sg. $\check{s} \underline{a}$, s. dort) 'Naht' (Sivić). Vgl. Skok III 396 f. s. v. šiti.

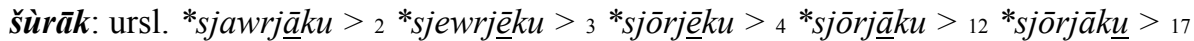

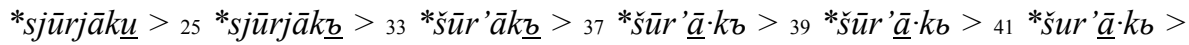

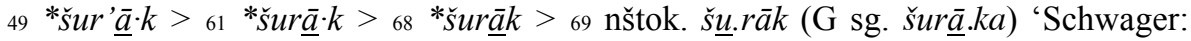
Bruder der Frau’ (Berlakovich). Vgl. ARj XVII 893, Trautmann 1970: 261, Skok III 424, Derksen 2008: 488 s. v. šurb. Zum Suffix vgl. jùnāk in Holzer 2007: 102. 


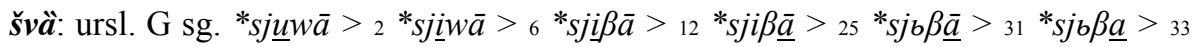

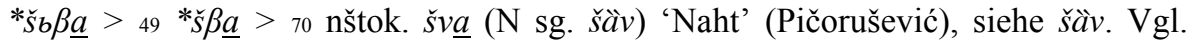
Skok III 396 f. s. v. šiti.

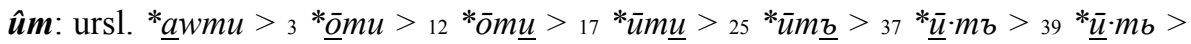
${ }_{49} * \underline{\bar{u}} \cdot m>68$ nštok. $\underline{\bar{u}} m$ (G sg. $\left.\underline{\bar{u}} . m a\right)$ 'Verstand' (Pičorušević). Vgl. Skok III 544, Snoj 2003: 798, Derksen 2008: $508 \mathrm{f}$.

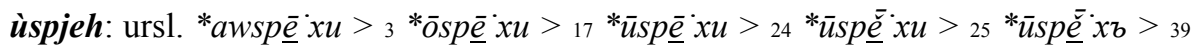

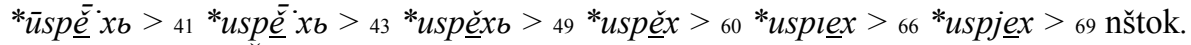
u.spjex 'Erfolg' (Šišić). Vgl. Skok III 427 (s. v. dòspjeti), Snoj 2003: 801.

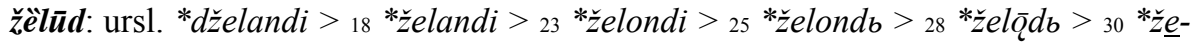
$l \bar{o} d b>{ }_{48} * \check{z} \underline{e} l \bar{u} d b>49$ nštok. že $\underline{e} \bar{u} d$ mask. 'Eichel' (Joksimović). Vgl. ARj XXIII 286, Skok III 675, Snoj 2003: 866, Derksen 2008: 556.

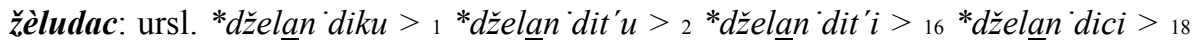

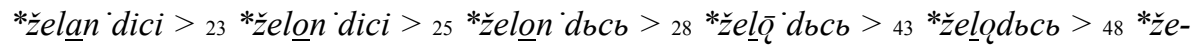
$\underline{l u} d b c b>{ }_{49} * \check{z} e \underline{e} \underline{u} d b c>{ }_{55} *_{z ̌} e l \underline{u} d a c>69$ nštok. že.ludac 'Magen' (Sivić). Nicht ver-

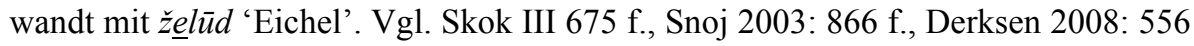
s. v. *želǫdzkz.

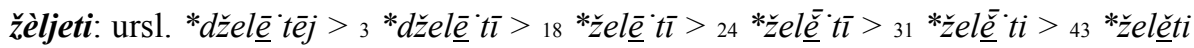

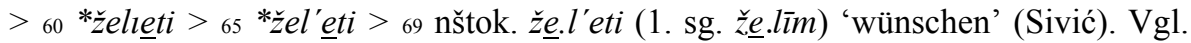
Skok III 676, Snoj 2003: 866, Derksen 2008: 555.

žèljezo: ursl. *džele $z a>{ }_{12} * d z ̌ e l \underline{e} z a$ (oder schon ursl. *dželēé $z a$ ) $>{ }_{18} * z ̌ e l \underline{e} z a>23$

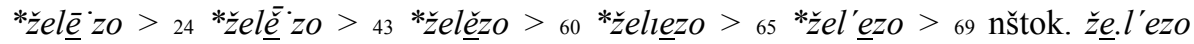
'Eisen' (D. Saraf). Vgl. Skok III 676 f., Snoj 2003: 866, Derksen 2008: 555 f.

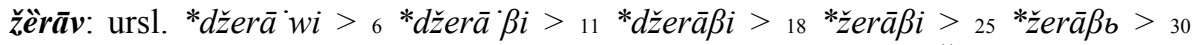
*žerā $\beta b>{ }_{49} * z ̌ e r a \bar{\beta} \beta>70$ nštok. žerāv (neben $\check{z} \underline{e} . r a v$ ) 'Kranich' (Šišić). Oder: ursl.

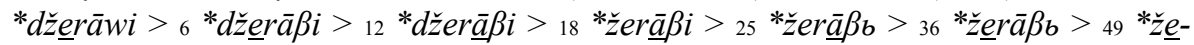
$r \bar{a} \beta>70$ nštok. žerāov. Vgl. ARj XXIII 337, Skok III 672 (s. v. ždrâl), Snoj 2003: 867 (akzentuiert žèrāv und rekonstruiert ursl. *žěrävb), Derksen 2008: 558.

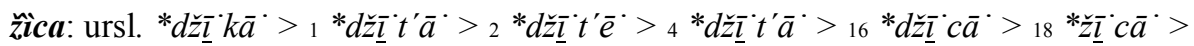
31 *žñ $c a>43$ nštok. žica 'Draht' (Đuričić). Vgl. Skok III 679 f., Snoj 2003: 869, Derksen 2008: $561 \mathrm{f}$.

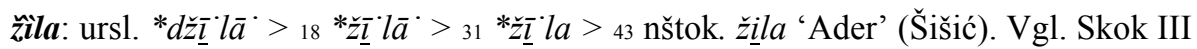
680, Snoj 2003: 869, Derksen 2008: 562.

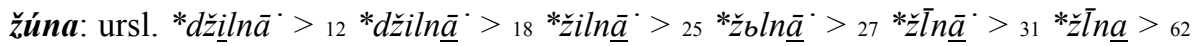
*žūna > 69 nštok. žzu. $n a$ 'Specht' (Šišić). Vgl. ARj XXIII 504, Skok III 686 f., Marko 
Snoj in Bezlaj IV 477, Snoj 2003: 873, Vukušić 2007: 663 (Akzentparadigma b), Derksen 2008: 565 (rekonstruiert Akut, aber kein Akzentparadigma).

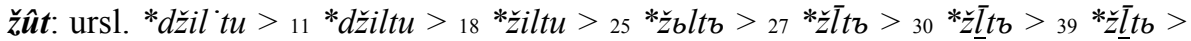
${ }_{49} * z ̌ \bar{l} \underline{l} t>62$ nštok. $z ̌ \bar{u} t$ (fem. žzu.ta) 'gelb' (Šišić). Vgl. Skok III 689, Snoj 2003: 873, Derksen 2008: 565 f.

\section{Li ter a tur}

ARj I-XXIII:

Bezlaj IV:

Derksen 2008:

Dybo 1981:

Holzer 2005:

Holzer 2007:

Holzer 2009:

Rječnik hrvatskoga ili srpskoga jezika. Na svijet izdaje Jugoslavenska akademija znanosti i umjetnosti I-XXIII, Zagreb 1881-1976

France Bezlaj, Etimološki slovar slovenskega jezika. Četrta knjiga ŠŽ. Avtorji gesel France Bezlaj, Marko Snoj in Metka Furlan. Uredila Marko Snoj in Metka Furlan, Ljubljana 2005

Rick Derksen, Etymological Dictionary of the Slavic Inherited Lexicon, Leiden - Boston

Vladimir Antonovič Dybo, Slavjanskaja akcentologija. Opyt rekonstrukcii sistemy akcentnych paradigm v praslavjanskom, Moskva

Georg Holzer, Zur relativen Datierung prosodischer Prozesse im Gemeinslavischen und frühen Kroatischen, Wiener Slavistisches Jahrbuch 51, 31-71

Georg Holzer, Historische Grammatik des Kroatischen. Einleitung und Lautgeschichte der Standardsprache (= Schriften über Sprachen und Texte 9), Frankfurt am Main u. a.

Holzer 2010:

Georg Holzer, Urslavische Prosodie, Wiener Slavistisches Jahrbuch $55,151-178$

Georg Holzer, Urslavische Morphophonologie. Ein Entwurf mit Beispielen aus der Slavia submersa Niederösterreichs, in: Elena StadnikHolzer und Georg Holzer (Hrsg.), Sprache und Leben der frühmittelalterlichen Slaven. Festschrift für Radoslav Katičić zum 80. Geburtstag. Mit den Beiträgen zu den Scheibbser Internationalen Sprachhistorischen Tagen II und weiteren Aufsätzen (= Schriften über Sprachen und Texte 10), Frankfurt am Main u. a., 43-77

Jurišić 1973: Blaž Jurišić, Rječnik govora otoka Vrgade uspoređen s nekim čakavskim i zapadnoštokavskim govorima. II. dio: rječnik, Zagreb

Kapović 2006: $\quad$ Mate Kapović, Naglasne paradigme $o$-osnova muškoga roda u hrvatskom, Rasprave Instituta za hrvatski jezik i jezikoslovlje 32, 159-172

Kortlandt 1975: $\quad$ F. H. H. Kortlandt, Slavic Accentuation. A Study in Relative Chronology, Lisse

Lukežić - Zubčić 2007: Iva Lukežić - Sanja Zubčić, Grobnički govor XX. stoljeća (gramatika i rječnik), Rijeka

Skok I-IV: Petar Skok, Etimologijski rječnik hrvatskoga ili srpskoga jezika I-IV, Zagreb 1971-1974

Snoj 2003: Marko Snoj, Slovenski etimološki slovar, 2. Auflage, Ljubljana

Trautmann 1970:

Vasmer 1979:

Vukušić 2007: Reinhold Trautmann, Baltisch-Slavisches Wörterbuch, 2. Auflage, Göttingen

Max Vasmer, Russisches etymologisches Wörterbuch II, Heidelberg Stjepan Vukušić - Ivan Zoričić - Marija Grasselli-Vukušić, Naglasak u hrvatskome književnom jeziku (= Velika hrvatska gramatika. Knjiga četvrta), Zagreb 
A bstract: A glossary of diachronic phonology of Neoštokavian. This paper is a supplement to the glossary in Holzer 2007: 90-144. Several Neoštokavian words are derived from their Proto-Slavic forms via the phonetic laws defined in Holzer 2007: 51-88.

K e y w o r d s : Neoštokavian, Proto-Slavic, phonetic laws

Georg Holzer

Institut für Slawistik der Universität Wien

Universitätscampus AAKH, Hof 3

Spitalgasse 2, 1090 Wien, Österreich georg.holzer@univie.ac.at
Saša Dobrić

Ramperstorffergasse 35/1

1050 Wien, Österreich

sasa.dobric@chello.at 